\title{
Unraveling the drift behaviour of the remarkable pulsar PSR B0826-34
}

\author{
Y. Gupta ${ }^{1}$, J. Gil², J. Kijak ${ }^{2}$, and M. Sendyk ${ }^{2}$ \\ 1 National Centre for Radio Astrophysics, TIFR, Pune University Campus, Pune 411007, India \\ e-mail: ygupta@ncra.tifr.res.in \\ 2 Institute of Astronomy, University of Zielona Gora, Lubuska 2, 65-265 Zielona Gora, Poland \\ Received 11 November 2003 / Accepted 22 June 2004
}

\begin{abstract}
Pulsars with drifting subpulses are thought to be an important key to unlocking the mystery of how radio pulsars work. We present new results from high sensitivity GMRT observations of PSR B0826-34 - a wide profile pulsar that exhibits an interesting but complicated drifting pattern. We provide a model to explain the observed subpulse drift properties of this pulsar, including the apparent reversals of the drift direction. In this model, PSR B0826-34 is close to being an aligned rotator. Using information about the polarization and frequency evolution of the pulse profile, we solve for the emission geometry of this pulsar and show that the angle between the rotation axis and the dipole magnetic axis is less than $5^{\circ}$. As a result, our line of sight samples a circular path that is entirely within the emission beam. We see evidence for as many as 6 to 7 drifting bands in the main pulse at $318 \mathrm{MHz}$, which are all part of a circulating system of about 15 spark-associated subpulse emission beams that form, upon averaging, one conal ring of the mean emission. We also see evidence for a second ring of emission, which becomes dominant at higher frequencies (above $1 \mathrm{GHz}$ ) due to the nature of the emission geometry. We model the subpulse drift behaviour of this pulsar in detail, providing quantitative treatments of the aliasing problem and various effects of geometry which play an important role. The observed drift rate is an aliased version of the true drift rate which is such that a subpulse drifts to the location of the adjacent subpulse (or a multiple thereof) in about one pulsar period. We show that small variations, of the order of $3-8 \%$, in the mean drift rate are then enough to explain the apparent reversals of drift direction seen in the data. We find the mean circulation time of the drift pattern to be significantly longer than the predictions of the original Ruderman \& Sutherland (1975) model and propose an explanation for this, which relates to modified models with temperature regulated partial ion flow in the polar vacuum gap. The small variations in drift rate are then explained by very small heating and cooling effects - less than $3500 \mathrm{~K}$ change in the $\sim 2.5 \times 10^{6} \mathrm{~K}$ surface temperature of the neutron star polar cap. From a detailed consideration of the variation of the mean subpulse separation across the main pulse window, we show that the circulating spark pattern is not centred around the dipole axis, but around a point much closer (within a degree or so) to the rotation axis. This is an indicator of the presence of a "local pole" corresponding to the non-dipolar magnetic fields that are expected to be present close to the neutron star surface. PSR B0826-34 thus provides a very rich and powerful system in which to explore important aspects of the physics of pulsar radio emission and neutron star magnetospheres.
\end{abstract}

Key words. stars: neutron - stars: pulsars: general - stars: pulsars: individual: PSR B0826-34

\section{Introduction}

Understanding how radio pulsars work remains a challenging unsolved puzzle in astrophysics. The phenomenon of drifting subpulses seen in some pulsars is widely thought to be an important key to understanding this mystery. This phenomenon, first reported by Drake \& Craft (1968), manifests itself in the most basic form as an organised phase change of subpulses occurring in successive pulses, resulting in the formation of one or more drift bands spanning the pulse envelope (Backer 1973; Rankin 1986). The basic concept is illustrated in Fig. 8 of Backer (1973). In any single pulse, there are typically two to three approximately equispaced subpulses, with a separation of $P_{2}$ degrees of longitude, whose typical values are $\sim 10^{\circ}-20^{\circ}$. The interval between recurrence of successive drift bands at a given pulse longitude is called $P_{3}$ and typical values range from 1 to 15 times $P_{1}$, the pulsar period. The intensity of the subpulses is systematically modulated as they move along the drift band. Usually, the intensity peaks in the middle of the pulse envelope and decreases towards the edges; however, in a few cases, the opposite trend is also seen (Taylor et al. 1975). The direction in which the subpulses drift - either leading to trailing edge of the pulse window with pulse number, or the other way around - is another important aspect. Though both kinds of drifting pulsars are known, for a given pulsar, the direction in which the subpulses drift is usually fixed. However, there are a few exceptional pulsars where both directions of drift are seen (though at different times).

In the standard phenomenology (e.g., Rankin 1986), cases of clear subpulse driftband patterns are usually seen in pulsars where the line of sight grazes along the edge of the radio emission beam. For pulsars where the line of sight has a more 
central traverse over the beam, the phenomenon can manifest itself as periodic intensity modulations of the subpulses at a given longitude, without the detection of any phase changes (Backer 1973). The observed periodicities related to patterns of drifting subpulses are usually found to be independent of radio frequency, thus excluding all frequency dependent plasma effects as plausible mechanisms of the drifting subpulse phenomenon. All the properties discussed above strongly suggest that this phenomenon is a manifestation of the circulation of subpulse-associated subbeams around the magnetic axis.

The drifting subpulse phenomenon finds a natural explanation in the model of Ruderman \& Sutherland (1975, RS75 hereafter). This model proposes the occurrence of discrete, localised spark discharges in a quasi-steady vacuum gap that forms just above the neutron star surface, as the seed activity that drives the radio emission mechanism. Each spark generates a stream of electron-positron plasma that flows out along the dipolar field lines and, at heights that are estimated to be of the order of several tens of stellar radii, produces a beam of subpulse-associated radio emission due to some, as yet unidentified, plasma radiation mechanism. Further, this model proposes that the plasma of each spark discharge inside the vacuum gap undergoes a systematic motion around the magnetic axis, due to the $\boldsymbol{E} \times \boldsymbol{B}$ drift. This, coupled with the rapidly fluctuating but rarely self-quenching nature of the sparks, leads naturally to the phenomenon of drifting subpulses. This drift rate is slower than the corotation rate and the sparks therefore lag behind with every rotation. In this picture, each drifting spark would correspond to one band of drifting subpulses. Since the spark plasma can only lag the pulsar rotation, the model predicts drift in one direction only, depending on the relative orientation of the rotation and magnetic axes and the observer's line of sight for the given pulsar. The RS75 model also makes quantitative predictions of the total circulation time of the drifting pattern for a pulsar, which, in principle, can be checked against observations. Further refinements of the RS75 model include the models of Cheng \& Ruderman (1980), Gil \& Sendyk (2000, hereafter GS00) and Gil et al. (2003, hereafter GMG03) which attempt to overcome some of the deficiencies and problems identified with the RS75 model. Other models that address the issue of subpulse drift are those of Allen \& Melrose (1983); Jones (1984); Kazbegi et al. (1996); Wright (2003).

However, there are some problems in the interpretation of the observations that make it difficult to compare with theoretical models. Often, there are complications of the drift rates not being constant and stable with time. Even when the drift rates (or value of $P_{3}$ ) are stable and can be determined reliably, it is not clear whether they are the real values or not, as the measured values can be modified due to the effect of aliasing. This effect can occur if the sampling rate (once per pulsar period) is too slow to track the same subpulse-associated subbeam of radiation in a sequence of pulses. Further, the total number of sparks, $N_{\text {sp }}$, needs to be known to ascertain the total circulation time of the pattern. In all this, the exact geometry of the observations needs to be known for correct interpretation of the results.

As a result, though there are about 40 pulsars with observations and measurements of the phenomenon of drifting subpulses (for a summary see Rankin 1986), there are only a few for which quantitative results have been obtained which can be compared against the predictions of the models. For PSR B0943+10, from a careful analysis of the fluctuation spectra, Deshpande \& Rankin (1999) were able to resolve the alias order for the observed periodicity $\left(P_{3}\right)$ and also infer the presence of 20 spark associated subpulse columns circulating with a time of $37 P_{1}$. For PSR B0809+74, van Leeuwen et al. (2003) have recently shown that the drift rate we see is an unaliased version and hence $P_{3}>150 P_{1}$ and $N_{\mathrm{sp}} \geq 14$. For both cases, the final circulation time turns out to be significantly longer than the prediction of the RS75 model. Modified versions of this model (e.g., GMG03) attempt to account for these discrepancies.

Whereas observations of pulsars with drifting subpulses where the line of sight just grazes a cone of emission produces a clear signal for analysis and interpretation, a similar phenomenon when seen for pulsars where the line of sight samples a large fraction of the conal ring can produce additional insight into the phenomenon. This makes pulsars with wide profiles very interesting. PSR B0826-34 is such a case. It has a very wide profile, showing emission over as much as $250^{\circ}$ of longitude at metre wavelengths (Durdin et al. 1979; Biggs et al. 1985; Reyes et al. 1995). However, it is not a widely studied pulsar, probably because of the fact that it nulls for as much as $70 \%$ of time (Durdin et al. 1979), making it a difficult case to observe. The study reported by Biggs et al. (1985) is the most exhaustive study published to date for this pulsar. They found that there is subpulse structure at almost all longitudes in the wide profile of PSR B0826-34, with remarkable patterns of drifting subpulses present over $200^{\circ}$ of longitudes. They noted that the drift rate shows large variations, including reversals of the direction of the drift - they concluded that this behaviour is inconsistent with the prevailing theoretical models for the phenomenon of drifting subpulses. From the integrated pulse profiles and the polarization data at 408 and $610 \mathrm{MHz}$, Biggs et al. (1985) concluded that PSR B0826-34 is likely to be a nearly aligned rotator, i.e. with rotation and magnetic axes almost parallel to each other, although their formal best fit values for the angle between the rotation and magnetic axes don't fully support this conclusion. However, in the morphological scheme of Rankin $(1983,1993)$ it is classified as "M" type pulsar, i.e. having a central line of sight which goes close to the magnetic pole and produces distinct core and conal emission components in the average profile. From the catalogue values of period $=1.8489 \mathrm{~s}$ and period derivative $=1.0 \times 10^{-15} \mathrm{ss}^{-1}$, the inferred values of the dipolar magnetic field and characteristic age for this pulsar are $1.4 \times 10^{12} \mathrm{G}$ and $3.0 \times 10^{7} \mathrm{yr}$, respectively.

It is clear from the above description that PSR B0826-34 is an enigmatic pulsar that has the potential to reveal vital clues about the pulsar emission mechanism and therefore deserves a closer and more detailed study. With this in aim, we have been carrying out high sensitivity observations of this pulsar with the Giant Metre-wave Radio Telescope (GMRT). The results from our initial observations and the interpretations thereof are reported in this paper. In Sect. 2, we describe the observations and the basic data reduction. Section 3 presents the main results 
PSR B0826-34
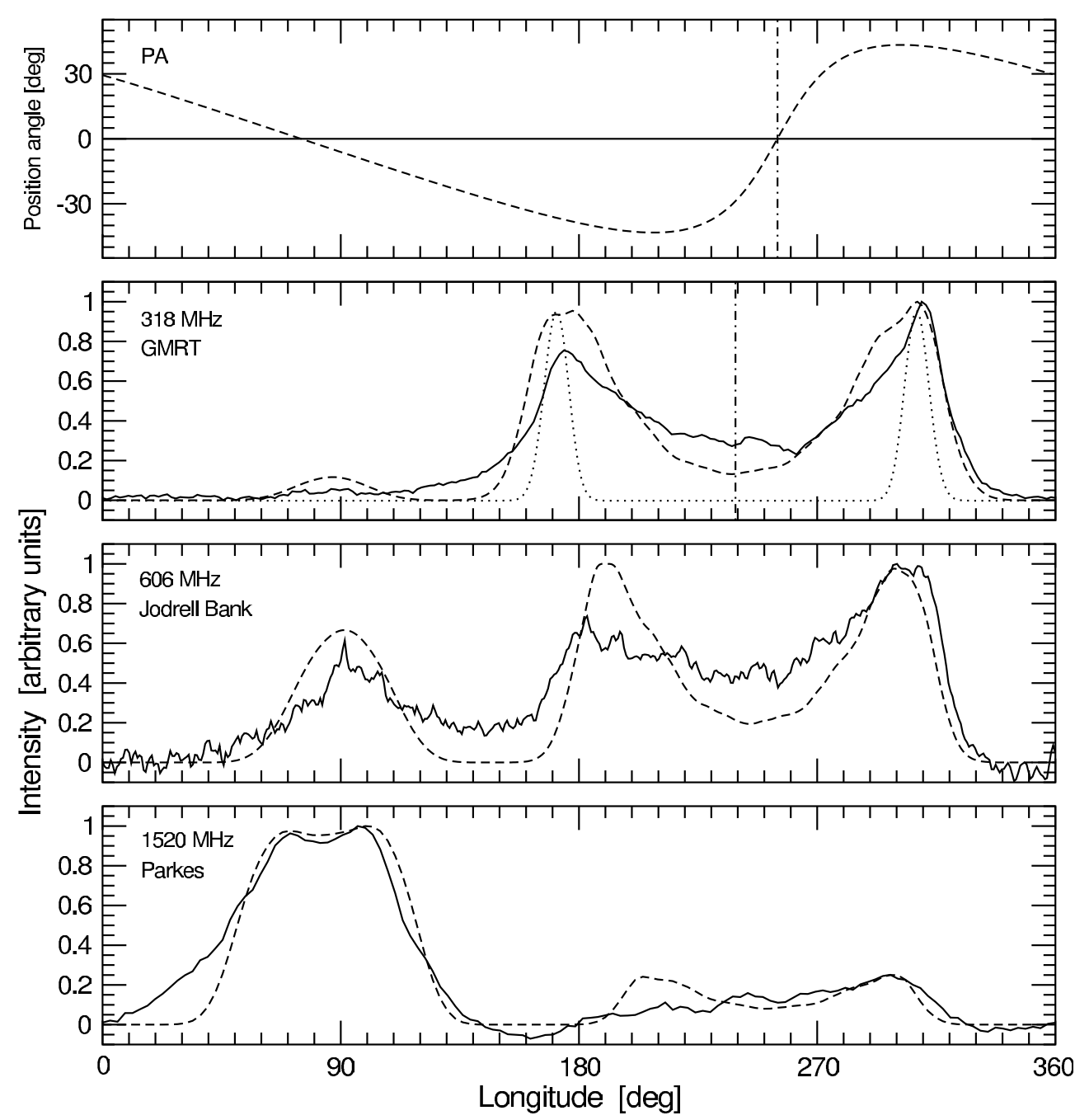

Fig. 1. Frequency evolution of the average profile of PSR B0826-34. The solid lines (bottom 3 panels) show the observed profiles at 3 different radio frequencies, including the result (at $318 \mathrm{MHz}$ ) from this paper. The dashed lines (bottom 3 panels) show the best fit simulated profiles corresponding to sparks circulating around the local surface magnetic pole, while the dotted line corresponds to spark circulation around the dipole axis (see text for explanations). The top panel shows the position angle curve corresponding to dipolar geometry with $\alpha=2.5$ and $\beta=1.0$. Note that the inflexion point of the position angle curve lags the midpoint of the main pulse by about $15^{\circ}$.

and our model that interprets these results, including determination of the emission geometry for this pulsar and detailed description of various effects that modify the true values of basic quantities related to the drifting phenomenon. In Sect. 4 we discuss the implications of different aspects of our model and present a final summary. In Appendix A we summarise the basic ideas relevant to the understanding of pulsar emission geometry, and in Appendix B we describe the details of our simulation studies.

\section{Observations and data analysis}

PSR B0826-34 was observed on Dec. 11, 2000 at the GMRT, in the $325 \mathrm{MHz}$ band, using the incoherent array mode (see Gupta et al. 2000, for more details about the pulsar modes of operation of the GMRT). The data were obtained by incoherent addition of the dual polarization signals from 13 antennas. The center frequency of the observations was $318 \mathrm{MHz}$. The bandwidth used was $16 \mathrm{MHz}$, divided into 256 spectral channels by the digital back-ends. The raw data were integrated to a time resolution of $0.516 \mathrm{~ms}$ before being recorded for off-line analysis, where they were further integrated to obtain a final time resolution of $4.128 \mathrm{~ms}$. The duration of the observation was about $22 \mathrm{~min}$, resulting in recorded data covering 714 periods of this pulsar. Of this, only the first 500 or so pulses were useful; the pulsar was in the null state after that, and no detectable signal could be obtained from the last 200 pulses.

During off-line analysis, the data were first dedispersed and then folded synchronously with the Doppler corrected pulsar period to obtain the average profile, which is shown in the second panel of Fig. 1. The first remarkable thing about the average profile for PSR B0826-34 is the unusually large pulse width : emission can be seen over more than $200^{\circ}$ of longitude. The "main pulse" has a double peaked structure with a component peak separation of $134^{\circ} \pm 4^{\circ}$, centred on the longitude of $240^{\circ}$. In addition, there is evidence for faint emission in the 
Table 1. Frequency dependence of component separation.

\begin{tabular}{rcl}
\hline \hline $\begin{array}{r}\text { Frequency } \\
(\mathrm{MHz})\end{array}$ & $\begin{array}{c}\text { Separation } \\
\text { (degrees) }\end{array}$ & References \\
\hline 610 & $112 \pm 4$ & Biggs et al. (1985) \\
408 & $128 \pm 4$ & Biggs et al. (1985); Durdin et al. (1979) \\
318 & $134 \pm 4$ & This paper \\
116 & $151 \pm 4$ & Turner (1980) \\
95 & $155 \pm 4$ & Turner (1980) \\
82 & $157 \pm 4$ & Turner (1980) \\
\hline
\end{tabular}

longitude range $55^{\circ}$ to $105^{\circ}$, which could be an interpulse, centred about $90^{\circ}$. These features are similar to what is seen in the $408 \mathrm{MHz}$ profiles of Biggs et al. (1985) and Durdin et al. (1979).

Comparing all the published average profiles available for this pulsar at different frequencies (Biggs et al. 1985; Durdin et al. 1979; Reyes et al. 1995, the EPN database), it is clear that there is a remarkable evolution of the pulse profile with frequency. It appears that the main pulse reduces in strength relative to the interpulse systematically, as one goes from lower to higher frequencies, to the extent that the interpulse actually dominates over the main pulse at frequencies above $\sim 1 \mathrm{GHz}$ (see lower 3 panels of Fig. 1, for example). Further, the component separation between the two peaks of the main pulse reduces with increasing frequency, following the general trend seen for a large majority of pulsars (referred to as the "radiusto-frequency mapping" model). In Table 1, we quantify this frequency evolution by compiling all the reported values for the component separation for the main pulse available for this pulsar, including our new results which are found to be compatible with the trend. The separation between the centres of the main pulse and the interpulse appears to be roughly frequency independent, at a value of about $150^{\circ}$ to $160^{\circ}$ (see Fig. 1).

As reported by Biggs et al. (1985), PSR B0826-34 exhibits remarkable subpulse structure in single pulse data. Figure 2 shows an example of single pulse data, covering the first 200 pulses from our observations. Individual subpulses can be seen over almost the entire main pulse window. Further, there is evidence for regions of organised drifting of the subpulses (in the form of linear drift-bands going from later to earlier longitudes with increasing pulse number - we refer to this as negative drift) over limited period ranges. There is also evidence for regions of oppositely oriented drift-bands (positive drift rate), as well as clear signs of curved drift-bands. In addition, there are sporadic, short duration nulls interspersed in the data. The over-all picture thus looks somewhat random and chaotic, and not very different qualitatively from what was reported by Biggs et al. (1985) for this pulsar at $645 \mathrm{MHz}$ (their Fig. 2).

However, the better signal-to-noise ratio of our data allows a clearer picture of the drifting subpulse pattern to emerge, from a gray-scale plot of the single pulse data (Fig. 3). Here, one can clearly see evidence for multiple drift-bands across the pulse window; 6-7 bands can be seen in most of the sections where the pulsar does not null. However, the drift behaviour

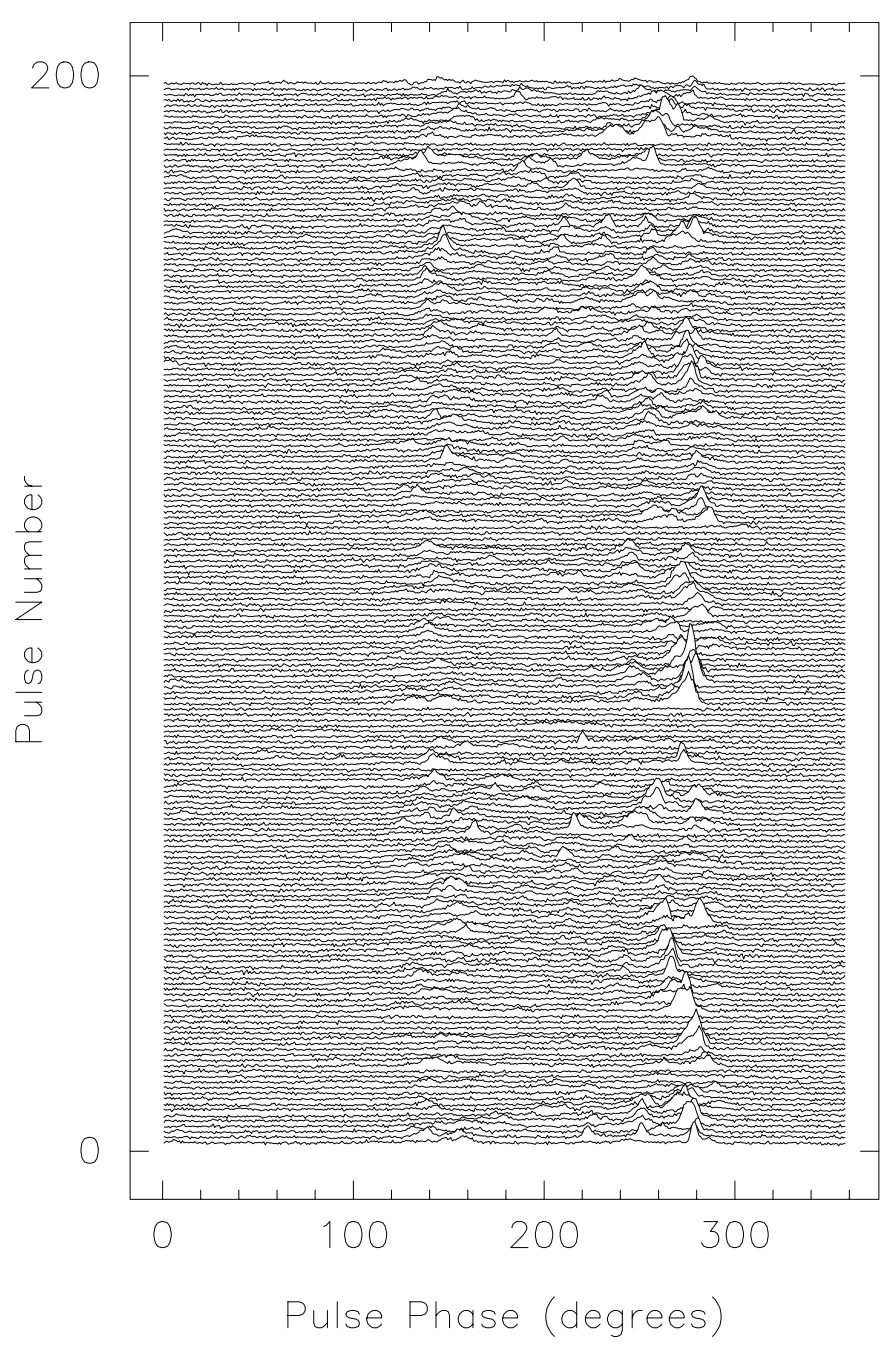

Fig. 2. Single pulse sequence of the first 200 pulses from the GMRT observations of PSR B0826-34 at $318 \mathrm{MHz}$.

of these bands of subpulses is not a regular, monotonic, linear drift pattern; instead it is much more irregular, exhibiting significant changes in the apparent drift rate, including change of sign. Nevertheless, the pulse regions showing drift rates of opposite sign are almost always connected by regions showing a smooth transition of the drift rate. This can clearly be seen for most cases where the apparent drift rate changes from negative to positive values, going smoothly through zero (e.g. see the regions of pulse \# 20 to 70 , pulse \# 120 to 180 and pulse \# 350 to 390). For the opposite transition (from positive to negative drift values), the change is usually more abrupt and not always easily traceable. Such regions (e.g. pulse \# 80 to 120 and pulse \# 180 to 220) correspond to the "run-away" sections identified by Biggs et al. (1985). The region of pulse \# 390 to 440 is an exception, where the complete transition can be traced. Therefore, in the most general case, the drift pattern for this pulsar consists of connected regions of drift-bands of opposite slopes. The typical values for the maximum positive and negative drift rates are $+1.9 /$ pulse (about $+1.0 / \mathrm{s}$ ) to $-0.8 /$ pulse (about $-0.4 / \mathrm{s}$ ). Further, the different curved sections of drift bands can be connected with a continuous curve, except for missing sections due to nulls. A typical time scale for 
one cycle of drift rate reversal is about 100 periods, and about 5 such cycles are present in the sequence of 500 pulses in Fig. 3. It is also very significant that the behaviour of all the visible drift bands is highly correlated, i.e. all of them simultaneously show the same kinds of changes in drift rate.

Since there are about 7 drift bands present in the main pulse region of about $160 \mathrm{deg}$, the typical separation between drift bands works out to about $23 \mathrm{deg}$. A quantitative estimate of the separation between adjacent drift-bands - traditionally referred to as $P_{2}-$ is obtained from the autocorrelation function of the single pulses, computed over the entire pulse window and averaged over all the available pulses. The result is shown in Fig. 4, where the secondary peak is due to the correlation between adjacent drift-bands. From the location of this peak, the average value of $P_{2}$ is found to be $24.9 \pm 0.8 \mathrm{deg}$, close to the rough estimate given above. This is somewhat smaller than the value of $29.0 \pm 2.0$ obtained by Biggs et al. (1985) at $645 \mathrm{MHz}$. In addition, there is some indication from our raw data itself (e.g. Fig. 3), of variation of the value of $P_{2}$ across the pulse window, with a tendency to increase from the centre to the edge of the main pulse. We return to this aspect in detail in Sect. 3.3.

\section{Results and interpretation}

To summarise the main results from our analysis: PSR B0826-34 has a unusually wide average profile and the single pulse data show multiple drifting bands of subpulses which drift in a highly correlated manner but show significant changes in the apparent drift rate, including reversals of the drift direction. Our basic model to explain this behaviour is as follows: this pulsar is an almost aligned rotator (i.e. dipole magnetic axis and rotation axis are almost aligned). As a result, our line of sight traces out a circular track through the emission beam, centred very close to the magnetic axis. This allows us to sample the radiation from a large number of subpulse-associated plasma columns present in one particular conal ring of the emission beam, resulting in the large number of drifting bands of subpulses that are seen. The subpulse-associated plasma columns are produced by an arrangement of spark discharges occurring in the vacuum gap above the polar cap, as envisaged in models such as in RS75 and GS00. The number of such sparks that need to be present in this particular ring, to explain the observed number of drift bands, is about 15 . These spark discharges circulate in closed tracks on the polar cap, presumably due to the $\boldsymbol{E} \times \boldsymbol{B}$ drift. Further, the observed drift rate of the these bands is not the intrinsic drift rate, but an aliased version. In this case, the intrinsic drift rate is such that during one rotation period, a subpulse drifts to reach the location of the adjacent subpulse, or a multiple thereof; i.e. $P_{3}$ - traditionally defined as the time interval required for the subpulse pattern to repeat at the same longitudes - has a value $\approx P_{1} / k$ (where $k$ is an integer and $P_{1}$ is the pulsar period). If the drift rate is such that $P_{3}=P_{1} / k$ exactly, then the observed drift-bands would be longitudinally stationary, i.e. zero apparent drift rate. Now in addition, if there are small fluctuations in the intrinsic drift rate around this mean value, then the slowing down and speeding up of the intrinsic drift rate would be seen as apparent drifts in opposite
PSR B0826-34 at $318 \mathrm{MHz}$ using the GMRT

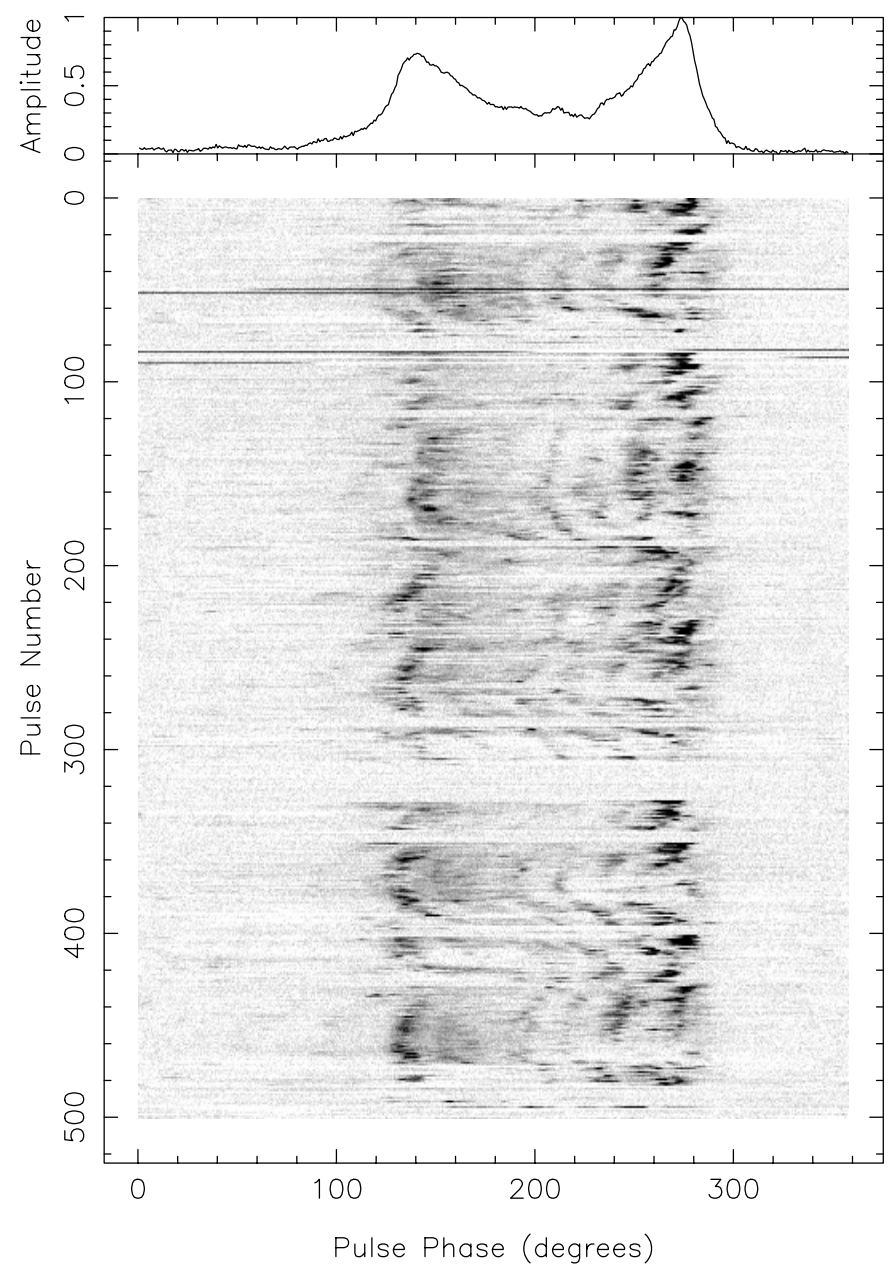

Fig. 3. Grey scale plot of single pulse data for 500 pulses of PSR B0826-34 from the GMRT observations at $318 \mathrm{MHz}$, with the average profile shown on top. The dark horizontal lines between pulse numbers 50, 60 and between 90, 100 are due to radio frequency interference.
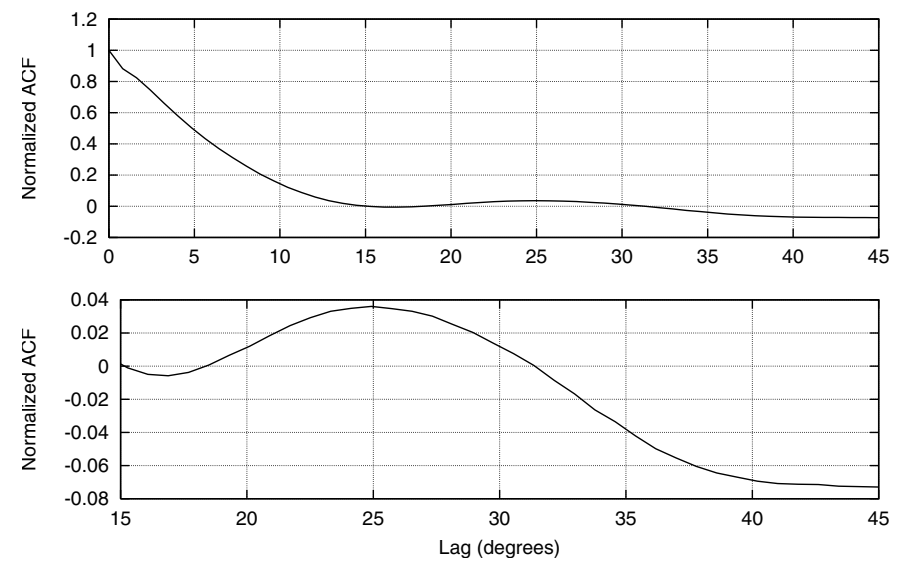

Fig. 4. Autocorrelation results for the entire on-pulse window data in Fig. 3. The lower panel shows a detailed view around the secondary maxima (near the lag of $25 \mathrm{deg}$ ) of the autocorrelation function.

directions. Such stroboscopic effects would then give rise to the apparent curved drift-bands that are observed in this pulsar. 
The small fluctuations of the drift rate could be produced by thermal effects on the polar cap that regulate the ion flow in the acceleration gap (Cheng \& Ruderman 1980, GMG03).

We now discuss different aspects of our model in detail. We start with a determination of the emission geometry for this pulsar, using information about its polarization properties and frequency evolution of the profile (Sect. 3.1). This is followed by a detailed modeling of the drift behaviour (Sect. 3.2), including the effect of aliasing and the effect of geometry on the estimation of $P_{2}$. In the light of these, we present our final interpretation of the data (Sect. 3.3), deriving the possible values for the various drift related parameters for this pulsar.

\subsection{Determination of the geometry}

Determination of the emission geometry is vital for proper interpretation of the results. The most important parameters here are the following: $\alpha$, the angle between the rotation and magnetic axes; $\beta$, the angle of closest approach between the magnetic axis and the line of sight (also called the impact parameter); and $\rho$, the radius of the emission beam which, in general, is a frequency dependent quantity. The beam radius can also be replaced by specifying the parameters $s$ (normalised distance from the dipole axis to the foot of the dipolar field line, a frequency independent quantity) and $r_{\mathrm{em}}$ (the emission height of the radio radiation, a frequency dependent quantity). The basic equations describing the relationships between these quantities are recapitulated in Appendix A.

Traditionally, the values of $\alpha$ and $\beta$ for a pulsar are determined from fits to the polarization angle (PA) curve of the pulsar, using the rotating vector model (Eq. (A.4)). However, as has been shown by several authors (Miller \& Hamilton 1993; van Ommen et al. 1997), there are problems in obtaining reliable, independent estimates of these angles from this procedure, in that a wide range of combination of values of $\alpha$ and $\beta$ are found to give acceptable fits. For the specific case of PSR B0826-34, Biggs et al. (1985) have shown that the polarization angle swing is compatible with a large range of choices of $\alpha$ and $\beta$. Furthermore, this method does not provide any information about the beam radius. The latter is usually estimated from a suitable measurement of the pulse width (such as separation between component peaks, $10 \%$ width etc.) and the use of Eq. (A.1), after the values for $\alpha$ and $\beta$ have been determined (see Appendix A).

To determine the emission geometry for PSR B0826-34, we have followed a slightly different technique which combines the available polarization information with the measured frequency evolution of the component separation, to constrain the values of the geometry parameters. Specifically, we use the following constraints: value of the steepest gradient of the PA curve, which we estimate to be $2.0 \pm 0.5 \mathrm{deg}$ per degree of longitude, from Fig. 6 of Biggs et al. (1985) (we note that Lyne \& Manchester (1988) quote $1.7 /^{\circ}$ for this value); the total swing of the polarization angle over the main pulse region, which we estimate to be $\approx 90^{\circ}$, again from Fig. 6 of Biggs et al. (1985); the measured values of the separation of the main pulse components, as listed in our Table 1 . We performed a grid search in values of $\alpha, \beta$ and $s$ to find all possible combinations of values that satisfy these constraints at all the frequencies listed in Table 1 (relevant details are described in Appendix A). Viable solutions for $\alpha$ and $\beta$ were found for values of the $s$ parameter in the range $0.25 \leq s \leq 0.85$. These solutions were in the range $1.5 \leq \alpha \leq 5.0$ and $0.6 \leq \beta \leq 2.0$, with the value of $\alpha$ greater than $\beta$ such that the requirement of Eq. (A.5) was satisfied. The extremes of the solution range were: $s=0.25$, $\alpha=1.5, \beta=0.6$ for the smallest sized conal ring to $s=0.85$, $\alpha=5.0, \beta=2.0$ for the largest sized conal ring. Further, from the typical S-shaped PA curve (Biggs et al. 1985, and also the upper panel in our Fig. 1), it is clear that we have a case of an "outer" line of sight, i.e. a case where the magnetic axis lies between the rotation axis and the line of sight, giving a positive value for $\beta$ (see Narayan \& Vivekanand 1982).

It is indeed remarkable that we are able to obtain such good fits matching the profile evolution over the frequency range of $82 \mathrm{MHz}$ to $610 \mathrm{MHz}$, while meeting the basic polarization angle requirements. Based on fits to polarization data only, Biggs et al. (1985) obtained very different values for $\alpha$ and $\beta$ for this pulsar. Using their values, it is impossible to reproduce the observed pulse widths for any realistic emission height model. In the allowed range of $0.25 \leq s \leq 0.85$, we tend to favour smaller values of $s(\approx 0.25$ to 0.5$)$, as we believe that there is another, outer conal ring of emission for this pulsar, with an $s$ value of $\approx 0.7$ (see Sect. 4). As a typical set of values, we use $s=0.44, \alpha=2.5, \beta=1.0$ wherever specific values are required in the rest of this paper. For this specific set of values of $s, \alpha$ and $\beta$, the predicted separation between the component peaks is $116^{\circ}, 124^{\circ}, 130^{\circ}, 152^{\circ}, 158^{\circ}$ and $159^{\circ}$ at frequencies of $610,408,318,116,95$ and $82 \mathrm{MHz}$, respectively, which agree very well with the measurements listed in Table 1 . We note that Lyne \& Manchester (1988) report best fit values of $\alpha=2.1, \beta=1.2$ (their Table 1) and Rankin (1993) reports $\alpha=3^{\circ}, \beta=1$. 1 , both of which are compatible with our results.

These results confirm our first hypothesis that we are looking at a pulsar that is very close to being an aligned rotator.

\subsection{Understanding the drift behaviour}

The basic observable quantities involved in understanding the subpulse drift pattern for a pulsar are illustrated in Fig. 8 of Backer (1973). We start our discussion with the idealized case of a perfectly aligned rotator $\left(\alpha=0^{\circ}\right)$, in which case the line of sight traces out a circle on the polar cap, centred exactly around the magnetic axis. Further, we assume that the line of sight is exactly coincident with a conal ring of emission. In such a case, regularly spaced subpulses would be visible throughout the pulsar period. Later we modify our discussion to include realistic cases. The principal periodicities that can be measured are $P_{1}, P_{2}, P_{3}$. The $P_{3}$ periodicity is often expressed in terms of a "fluctuation frequency", $f_{3} \equiv 1 / P_{3}$. These can be related to the drift rate, $D$, as follows:

$f_{3}=D / P_{2}=D_{\mathrm{p}} /\left(P_{1} P_{2}\right)$,

where $P_{1}$ and $P_{3}$ are in units of seconds ( $f_{3}$ is in $\mathrm{Hz}$ ), $P_{2}$ is in degrees, $D$ is in degrees per second and $D_{\mathrm{p}}$ is in degrees per 
period. If there are $N_{\mathrm{sp}}$ equispaced sparks in a circular ring on the polar cap, then

$P_{2}=\frac{360^{\circ}}{N_{\mathrm{sp}}}$.

Further, the total circulation time of the pattern, i.e. the time it would take a drifting spark to complete one full circulation, can be expressed as

$P_{4}=N_{\text {sp }} P_{3}=\frac{360^{\circ}}{D}$

where $P_{4}$ is measured in seconds. Thus, in principle, the measured values of $P_{1}, P_{2}$ and $P_{3}$ can be used to find the drift rate and the total circulation time of the spark pattern, which can be compared with the predictions of theoretical models.

In practice, however, the situation is complicated by the fact that the measured values of $P_{2}$ and $P_{3}\left(P_{2}^{\mathrm{m}}\right.$ and $\left.P_{3}^{\mathrm{m}}\right)$ need not correspond to the true values $\left(P_{2}^{\mathrm{t}}\right.$ and $\left.P_{3}^{\mathrm{t}}\right) ; P_{2}$ can be affected by the drift rate and by the viewing geometry, and $P_{3}$ can be affected due to aliasing. Also, some of the above equations are not valid for the general case of the non-aligned rotator. We first consider the effect of aliasing on $P_{3}$ and then examine the factors affecting $P_{2}$ and the effect of the viewing geometry.

\subsubsection{Effect of aliasing on drift behaviour}

Since the signal at any given pulse longitude is sampled only once per pulsar rotation period, the interpretation of any periodic signal measured at that longitude (such as the periodicity represented by $P_{3}$ ) is subject to the constraints of this sampling rate. For sufficiently slow drift rates (such that $P_{3}^{\mathrm{t}}>2 P_{1}$, or $f_{3}^{\mathrm{t}}<$ $\left.0.5 / P_{1}\right), P_{3}^{\mathrm{m}}$ is the same as $P_{3}^{\mathrm{t}}$. For $P_{3}^{\mathrm{t}}=2 P_{1}$, a subpulse drifts to the longitude of the adjacent subpulse in exactly 2 periods. This corresponds to the Nyquist periodicity, with $f_{\mathrm{N}}=0.5 / P_{1}$ being the Nyquist frequency. For faster drift rates (such that $P_{3}^{\mathrm{t}}<2 P_{1}$, or $f_{3}^{\mathrm{t}}>f_{\mathrm{N}}$ ), an aliased version of the periodicity is seen. For $P_{3}^{\mathrm{t}}$ slightly less than $2 P_{1}, P_{3}^{\mathrm{m}}$ is slightly more than $2 P_{1}$ and the apparent drift is of opposite sign than the true drift. As $P_{3}^{\mathrm{t}}$ reduces further, $P_{3}^{\mathrm{m}}$ keeps increasing correspondingly, till $P_{3}^{\mathrm{t}}=P_{1}$, at which point $P_{3}^{\mathrm{m}}$ becomes infinite $\left(f_{3}^{\mathrm{t}}=2 f_{\mathrm{N}}, f_{3}^{\mathrm{m}}=\right.$ $0)$ - this corresponds to the case of longitude stationary subpulse patterns, with zero apparent drift. As $P_{3}^{\mathrm{t}}$ reduces further $\left(f_{3}^{\mathrm{t}} \geq 2 f_{\mathrm{N}}\right), f_{3}^{\mathrm{m}}$ begins to increase and a non-zero drift rate is again measurable; however, the direction of the drift is now the same as the true direction. In fact, the apparent drift direction reverses sign every time the true drift rate crosses a multiple of the Nyquist boundary $\left(n f_{\mathrm{N}}\right)$, whereas the apparent drift amplitude goes through zero for every "even" crossing ( $n=$ even number) and through the maximum observable value for every "odd" crossing ( $n=$ odd number). In Appendix B we present results from simulations which illustrate some of these effects.

Quantitatively, the above behaviour can be described as follows:

$f_{3}^{\mathrm{t}}=2 k f_{\mathrm{N}}+(-1)^{l} f_{3}^{\mathrm{m}}, \quad f_{3}^{\mathrm{m}}=\frac{D^{\mathrm{m}}}{P_{2}^{\mathrm{m}}} ;$

where $k=\operatorname{INT}[(n+1) / 2]$ and $l=\bmod (n, 2)$, and $n$ is the alias order such that $n f_{\mathrm{N}}<f_{3}^{\mathrm{t}}<(n+1) f_{\mathrm{N}}$. This can be considered as a generalised form of Eq. (1), which special case obtains for $n=0$.

Thus, in order to estimate the true value of $P_{3}$ (and hence determine $N_{\mathrm{sp}}$ and $P_{4}$ in accordance with Eq. (3)) the alias order, $n$, has to be resolved; only for $n=0$ is there a one to one correspondence between the measured and true quantities. In general, it is not always possible to infer the value of $n$ from the observations. As described in Sect. 1, it has been achieved for only 2 pulsars - PSR B0943+10 (Deshpande \& Rankin 1999) and PSR B0809+74 (van Leeuwen et al. 2003) - using extra information from specific features present in the data.

For PSR B0826-34, our proposal is that we are seeing an aliased drift rate such that $n=2,4,6 \ldots$ (i.e. $k=1,2,3 \ldots$ ) and the overall mean drift rate is such that $f_{3}^{\mathrm{m}} \approx 0$. Thus $f_{3}^{\mathrm{t}} \approx 2 k f_{\mathrm{N}}$. In addition, there are small variations in $f_{3}^{\mathrm{t}}$ with an amplitude $\left|f_{3}^{\mathrm{m}}\right| \ll f_{\mathrm{N}}$, but large enough such that it crosses back and forth across the Nyquist boundary ( $n$ goes to $n-1, k$ remains the same, $l$ changes sign), producing the observed pattern with apparent reversals of drift direction.

The effect of aliasing also complicates the effort to resolve the direction of the true drift and compare it with model predictions. In vacuum gap models, the $\boldsymbol{E} \times \boldsymbol{B}$ drift of the spark plasma in the gap is slower than the corotation speed, i.e. the sparks lag behind with respect to the rotation of the star (e.g., RS75). The direction of the measured subpulse drift that this translates to depends on the line of sight: for an "inner" line of sight (negative $\beta$ ), the subpulses drift from the trailing edge to the leading edge of the pulse window with increasing pulse number, i.e. negative drift; for an "outer" line of sight (positive $\beta$ ), the drift is from leading to trailing (positive drift). However, for a case where $\alpha$ is very small, such that the spin axis lies within the emission cone $(\alpha \leq \rho)$, there is only one direction of drift for any kind of line of sight: drift from leading to trailing (positive drift) - see Ruderman (1976) for similar arguments. Of course, the final observed direction of the drift is further modified by the aliasing effect as described earlier in this section.

For PSR B0826-34, we have shown that we are dealing with an almost aligned rotator, with values of $\alpha$ that are smaller than the angular size of the ring of sparks (Sect. 3.1). Further, we are quite certain that we are dealing with an "outer" line of sight geometry. Both these factors imply that the intrinsic drift rate should be positive (i.e. drift from leading to trailing in the pulse window). For an unaliased drift, the direction of the apparent drift will be same as that of the intrinsic drift. As shown earlier, the apparent drift reverses direction each time the amplitude of the intrinsic drift rate crosses a multiple of the Nyquist boundary. Now, in our model the intrinsic drift rate for PSR B0826-34 is such that we are close to an even Nyquist ${ }^{1}$ boundary $(n=2,4,6 \ldots)$, with small variations around this value. When the intrinsic drift rate is slightly above the

\footnotetext{
1 In fact, for odd Nyquist boundaries $(n=1,3,5, \ldots)$ one would observe clear "even-odd" intensity modulations like in PSR B0943+10 (Deshpande \& Rankin 1999; GS00, their Fig. 2) and PSR B2303+30 (GS00, their Fig. 3), which are, however, not observed in PSR B0826-34 (see Appendix B for further discussion of aliasing effects).
} 


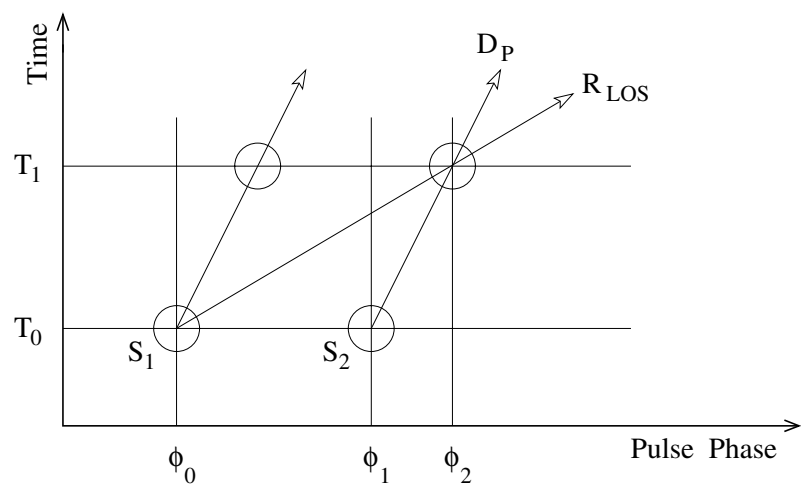

Fig. 5. Illustration of the concept of drift induced modification of $P_{2}$. $S_{1}$ and $S_{2}$ are two sparks located at longitudes $\phi_{0}$ and $\phi_{1}$ at time $T_{0}$ (i.e. $P_{2}^{\mathrm{t}}=\phi_{1}-\phi_{0}$ ). At $T_{0}$, the line of sight (moving along $R_{\mathrm{LOS}}$ ) is at $\phi_{0}$, and sees $S_{1}$. At a later time $T_{1}$, the line of sight is at $\phi_{2}$, where it sees spark $S_{2}$. Hence $P_{2}^{\mathrm{m}}=\phi_{2}-\phi_{0}$. During the interval $T_{1}-T_{0}$, the sparks (moving along $D_{P}$ ) drift by an amount $\phi_{2}-\phi_{1}$.

Nyquist boundary, the apparent drift direction should be same as the intrinsic. This means that the regions of positive drift in Fig. 3 are regions where the drift rate is slightly faster than the mean rate and regions of negative drift are regions where the drift pattern slows down somewhat. Coupling this with the drift behaviour described in Sect. 2, we conclude that the transition when the drift rate speeds up from less than the mean value to over the mean value is a relatively smoother and slower process than the opposite change when the drift rate slows down, which is more abrupt and faster. The implications of this are discussed further in Sect. 4.

\subsubsection{Effect of geometry on $P_{2}$}

The measured value $P_{2}^{\mathrm{m}}$ does not correspond to the true value $P_{2}^{\mathrm{t}}$, due to two effects. The first is the effect of the finite time taken by the line of sight to traverse the beam. As the line of sight moves from one subpulse longitude to the next one, the subpulse pattern drifts in longitude by a small amount; as a consequence, the measured value $P_{2}^{\mathrm{m}}$ is a "Doppler-shifted" version of the actual subpulse separation. This is illustrated schematically in Fig. 5. It is straightforward to show that $P_{2}^{\mathrm{t}}$ and $P_{2}^{\mathrm{m}}$ are related by

$P_{2}^{\mathrm{t}}=P_{2}^{\mathrm{m}}\left(1-D_{\mathrm{p}}^{\mathrm{t}} / 360^{\circ}\right)$

where, in keeping with our convention, the sign of $D_{\mathrm{p}}^{\mathrm{t}}$ is positive for drift from leading to trailing longitudes (i.e. along the direction of traverse of the line of sight), and negative for the other direction. This modified value of $P_{2}$ needs to be used in Eqs. (1) and (2) above. In our model for PSR B0826-34, taking the slowest drift rate (corresponding to the alias order of $n=2$ or $k=1$ ) and the mean value of $P_{2}^{\mathrm{m}}$ found in Sect. 2, this correction amounts to about $7 \%$, and increases by the same amount for every increment in the value of $k$.

Finally, for the general case of the non-aligned rotator, the direct relation between $P_{2}$ and the angular spacing of the sparks on the polar cap (Eq. (2)) is no longer valid. This is because equal longitude intervals along the line of sight (which are

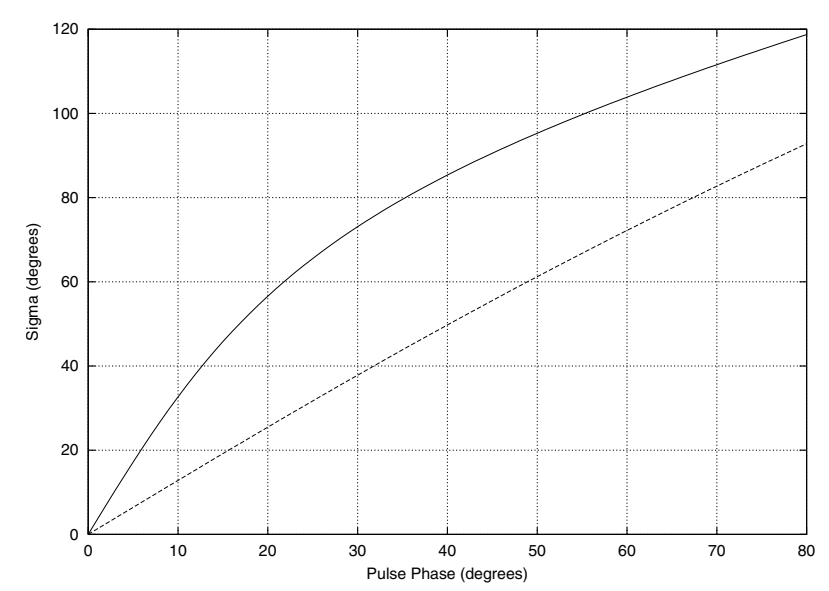

Fig. 6. Variation of $\sigma$ with $\phi$ (see Eq. (6) of text) for two different choices of geometry: $\alpha, \beta=2.5,1.0$ (solid curve) and $\alpha, \beta=0.8,2.8$ (dashed curve). The phase $\phi=0^{\circ}$ corresponds to the fiducial phase.

measured with respect to the rotation axis) do not map to equal angular intervals (magnetic azimuth) of the corresponding events on the polar cap ring (which are measured with respect to the magnetic axis). The general formula that relates azimuthal angles measured with respect to the magnetic axis $(\sigma)$ with the rotational phase $(\phi)$ is

$\sin (\sigma)=\frac{\sin (\alpha+\beta) \sin (\phi)}{\sin (\Gamma)}$

where $\Gamma$, the angle between the magnetic axis and the line of sight at the rotational phase $\phi$, is determined from Eq. (A.1). Figure 6 shows the variation of $\sigma$ as a function of $\phi$ for a couple of sample cases of emission geometry that are relevant for PSR B0826-34.

As a consequence of this effect, Eq. (2) can not be used as it stands to compute $N_{\mathrm{sp}}$. Instead, from a measurement of the longitudes of the subpulses, $\phi_{i}$ (or of the intervals between them, $\Delta \phi_{i}=P_{2}^{\mathrm{m}}$ ), one first needs to correct for the effect of scaling due to drift (Eq. (5)) and then map these corrected angles (or the intervals between them) to corresponding angular values $\left(\sigma_{i}\right.$ or $\left.\Delta \sigma_{i}\right)$ on the polar cap ring. For the correct solution, the values of $\sigma_{i}$ will be equispaced, and this constant interval will be

$\Delta \sigma=\frac{360^{\circ}}{N_{\mathrm{sp}}}$

provided that the subpulse-associated subbeams circulate around the magnetic dipole axis.

The conclusions from this section on understanding the drift behaviour can be summarised as follows. In order to estimate the total circulation time $\left(P_{4}\right)$, values of $P_{3}^{\mathrm{t}}$ and $N_{\mathrm{sp}}$ need to be estimated (Eq. (3)). The former requires knowledge of the alias order (Eq. (4)), and the latter requires knowledge of $P_{2}^{\mathrm{t}}$ (Eq. (2)). $P_{2}^{\mathrm{t}}$ can be estimated from the variation of $P_{2}^{\mathrm{m}}$ (Eqs. (5), (6) and (A.1)), provided the geometrical parameters $(\alpha, \beta)$ are known and the drift rate is known, which in turn requires knowledge of $P_{3}^{\mathrm{t}}$ and $P_{2}^{\mathrm{t}}$. Clearly, these equations form a coupled set which can not be solved directly to obtain the values of $N_{\mathrm{sp}}, n$ (and hence of $P_{3}$ and $P_{4}$ ); instead, some kind 
of iterative or grid search method is needed to find the allowed solutions.

\subsection{Interpretation of the data}

We now return to a closer inspection of our single pulse data to see what it can tell us about the nature of the variation of $P_{2}$ across the main pulse window. The correlation analysis that was used to estimated the mean value of $P_{2}^{\mathrm{m}}$ (as discussed in Sect. 2) was repeated over several narrow windows of pulse longitude (of about $40 \mathrm{deg}$ width), which were shifted systematically across the entire main pulse window (in steps of about $4 \mathrm{deg}$ ). For each case, the secondary peak of the correlation function was computed (where possible), providing an estimate of the value of $P_{2}^{\mathrm{m}}$ in that longitude range. Figure 7 shows sample plots of the correlation functions for 5 different windows, exhibiting significantly different values of $P_{2}^{\mathrm{m}}$. Figure 8 (top panel) shows the variation of $P_{2}^{\mathrm{m}}$ with pulse longitude, compiled from this correlation analysis. The missing data points correspond to cases where the correlation function did not show a clear secondary peak - these are regions of the pulse window where the drifting subpulse signal is relatively weak. The error bars for the $P_{2}^{\mathrm{m}}$ estimates are not from any rigorous calculations or curve fitting; they are estimates by eye, based on the quality (level of noise and the shape of the curve around the secondary maxima) of the cross correlation functions.

As seen in the top panel of Fig. 8, there appears to be a smooth trend for the variation of $P_{2}^{\mathrm{m}}$ across the pulse window, except for a few longitudes where there is a sudden, sharp increase in the estimated value. These outliers (four are clearly identifiable in the figure) are not well understood and are most likely due to the window spanning across some pairs of very strong subpulses associated with some of the drift-bands; we ignore these four data points in the subsequent analysis. The figure shows that there is a measurable variation of $P_{2}^{\mathrm{m}}$ across the main pulse window, which can be seen more clearly in the trailing half of the profile. The range of variation is from a minimum of about 21.5 to a maximum of $27^{\circ}$, and is compatible with the mean value of $24^{\circ} .9$ that we obtained, in Sect. 2, from the correlation function for the entire main pulse. The other interesting thing is that the minimum is not centred exactly in the middle of the on-pulse window, but appears at somewhat earlier longitudes, by about $15^{\circ}$ or so (though the large error bars make it difficult to come to very firm conclusions about this).

We now ask the question: can our inferred geometry (as derived in Sect. 3.1) explain this observed dependence of $P_{2}^{\mathrm{m}}$ with longitude? The solid line in Fig. 6 shows the variation of $\sigma$ with $\phi$ for the case of the $\alpha, \beta=2.5,1^{\circ} .0$, which is in middle of the solution range found in Sect. 3.1. It is clear from this plot that the variation of $\sigma$ is too fast for smaller values of $\phi$ and will lead to an over-correction of the smaller $P_{2}^{\mathrm{m}}$ values (in the middle of the pulse). This is true even after the measured subpulse longitudes are scaled to accommodate the effects of drift as described by Eq. (5). In fact, all the solutions found in Sect. 3.1 yield geometries where the line of sight is too "shallow" with respect to the emission ring, i.e. it is not sufficiently concentric with the emission ring. This is primarily because the value of $\alpha$

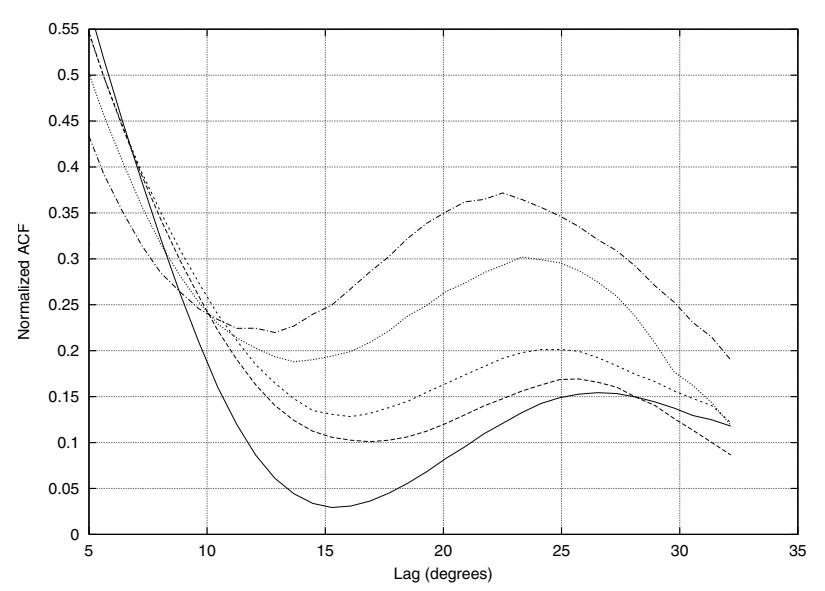

Fig. 7. Sample results of the autocorrelation function for narrow pulse longitude windows of the data in Fig. 3. Curves for 5 different window locations, centred at $269^{\circ}$ (solid), $253^{\circ}$ (long dashes), $245^{\circ}$ (short dashes), $229^{\circ}$ (dots) and $213^{\circ}$ (dash-dot) of pulse longitude are shown to illustrate the variation of $P_{2}^{\mathrm{m}}$ with longitude.

is too large, relative to that of $\beta$. For such geometries, not only is the observed variation of $P_{2}^{\mathrm{m}}$ across the pulse window incompatible with the predictions, the observed intensity levels in the saddle region of the main pulse are also much higher than the predictions, i.e. the central bands of drifting subpulses should be much weaker than what is observed. This is illustrated by the dotted-line simulated profile in Fig. 1 (see Appendix B for a description of the simulation technique), which is obviously inconsistent with the observed $318 \mathrm{MHz}$ profile - the components are too narrow and the saddle is too weak.

To explain the apparent disagreement between the emission geometry and the observed detailed behaviour of the subpulse pattern, we propose the following refinement of our basic model. Since the emission geometry constraints from pulse width and polarization data (Sect. 3.1) basically depend on the dipole field geometry at significant heights (roughly in the range 10-50 stellar radii), whereas the detailed variations of the subpulse structure are determined by events very close to the neutron star surface (in the "vacuum gap"), we propose that the centre of activity of these two are associated with different locations in the magnetosphere. More specifically, we propose that the spark pattern is not centred around the dipolar axis of the pulsar, but instead, circulates around some other point on the polar cap. In models which propose higher order or "multi-polar" magnetic field structure very near to the neutron star surface (e.g., Gil et al. 2002, and references therein), this alternate point of circulation could be the location of the "local magnetic pole" which, in general, could be offset from the dipole magnetic pole. In such a scenario, the generation of the spark pattern in the vacuum gap region is controlled by the local magnetic pole, with concentric rings of sparks being formed and circulating around this local pole. The subpulse-associated plasma columns generated by this activity then stream out into the magnetosphere along the dipolar field lines, which dominate the magnetic field structure above a certain height (certainly in the regions where we believe the radiation to originate). The location of the local pole (offset from the dipole) can 

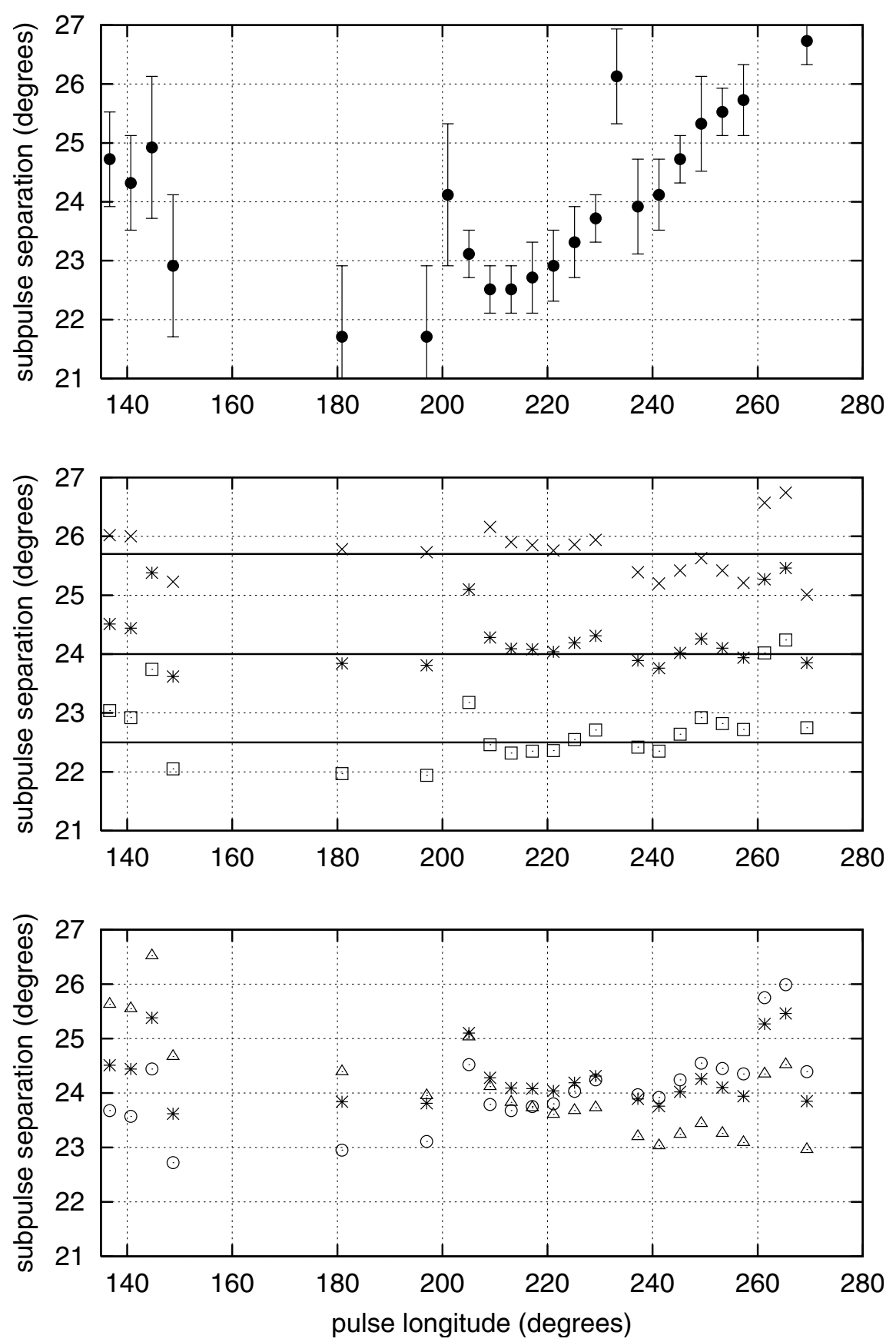

Fig. 8. Top panel: measured variation of $P_{2}^{\mathrm{m}}$ with longitude. Middle panel: inferred $\Delta \eta$ values as a function of pulse longitude, for each of the solutions list in Table 2; the 3 horizontal solid lines are the expected values $\left(360^{\circ} / N_{\mathrm{sp}}\right)$ for each case. Bottom panel: $\Delta \eta$ values for 3 cases of $N_{\text {sp }}, k=15,2$ solution, with $\phi_{\text {off }}=18^{\circ}$ (asterisks), $\phi_{\text {off }}=28^{\circ}$ (triangles) and $\phi_{\text {off }}=8^{\circ}$ (circles).

be specified in terms of a new set of angles $\alpha^{\prime}$ and $\beta^{\prime}$ analogous to $\alpha$ and $\beta$ (note that $\alpha^{\prime}+\beta^{\prime}=\alpha+\beta$ needs to be satisfied), and a rotational angle specifying the rotational phase difference $\phi_{\text {off }}$ between the fiducial plane containing the rotation axis and the dipole axis and the fiducial plane containing the rotation axis and the local pole axis.

From the variation of $P_{2}^{\mathrm{m}}$ (Fig. 8, top panel) and the model curves in Fig. 6, it is clear that in our model for PSR B0826-34, the local pole needs to be located significantly closer to the rotation axis than the dipole axis. Further, a rotational phase difference of about $15^{\circ}$ is suggested by the location of the minimum of the $P_{2}^{\mathrm{m}}$ curve in the top panel of Fig. 8. How can we make a more quantitative determination of the local pole geometry? We need simultaneous solutions for the following: values of $\alpha^{\prime}, \beta^{\prime}$ and $\phi_{\text {off }}$ for the local pole, value of $N_{\text {sp }}$ and the alias order $k$, such that the inferred values of angular separation of the sparks circulating around the local pole (we will use $\eta$ to refer to these angles, to differentiate from $\sigma$ used for angles with respect to the dipole axis), will satisfy the corresponding version of Eq. (7). To achieve this, a grid search in the values of $N_{\mathrm{sp}}, k, \alpha^{\prime}$ and $\beta^{\prime}$ was carried out. For each possible solution point in the grid search, a few different choices of $\phi_{\text {off }}$ were 
tried. For each combination, the $P_{2}^{\mathrm{m}}$ curve (top panel in Fig. 8) was "corrected" to obtain a curve of $\Delta \eta$ versus longitude (lower panels of Fig. 8) as follows. The measured $P_{2}^{\mathrm{m}}$ values at different phase locations $\left(\phi_{i}\right)$ were converted to a pair of individual phase locations $\left(\phi_{i} \pm 0.5 P_{2}^{\mathrm{m}}+\phi_{\text {off }}\right)$, and a correction for scaling due to drift was applied; these were then mapped to a pair of $\eta$ values (for the specified geometry) and their difference converted to a $\Delta \eta$ value for that $\phi_{i}$. Solutions that gave $\Delta \eta$ close to $360^{\circ} / N_{\mathrm{sp}}$ across the entire pulse window were taken as acceptable solutions. The range of values of $N_{\mathrm{sp}}, k$ tried was 14,1 to 17,4 and for $\alpha^{\prime}$ and $\beta^{\prime}$ it was 0 to $2.2 \mathrm{deg}$ and 1.0 to $7.0 \mathrm{deg}$, respectively.

The results from this analysis are summarized in Table 2, which lists various parameters for the different solutions. Acceptable solutions could be found for each choice of $N_{\mathrm{sp}}$. Of these, physically meaningful solutions (see Sect. 4 for a detailed explanation of this) are those for $N_{\mathrm{sp}}=14,15$ and 16 , and only these are included in Table 2. From Col. 2 of this table, it is seen that as the number of sparks is increased, the solution requires a higher value of alias order $k$ (i.e. a larger value of drift rate). This is because the smaller $P_{2}^{\mathrm{t}}$ from a larger $N_{\text {sp }}$ requires a faster drift so that the $P_{2}^{\mathrm{t}}$ values can be "stretched" to match the range of observed $P_{2}^{\mathrm{m}}$ values. For every such combination of $N_{\mathrm{sp}}, k$ values, there is small range of $\alpha^{\prime}, \beta^{\prime}$ values that result in equally acceptable solutions, in that they yield curves for $\Delta \eta$ versus longitude that are very similar, and have a mean value, $\Delta \eta_{\text {avg }}$, that is close to the expected value, $\Delta \eta_{\text {exp }}=360^{\circ} / N_{\text {sp }}$. This range is $\alpha^{\prime}, \beta^{\prime} \sim 0^{\circ} .4,2^{\circ} .0$ to 1.2 , 5.2 with $\alpha^{\prime}<\beta^{\prime}$ always - this is in contrast to the case of the dipole solutions obtained in Sect. 3.1, where $\alpha>\beta$. However, it is quite interesting to note that given the requirement $\alpha^{\prime}+$ $\beta^{\prime}=\alpha+\beta$, it is possible to find a combination of $\alpha^{\prime}$ and $\beta^{\prime}$ to match almost the entire range of dipole solutions in Sect. 3.1. Column 3 of Table 2 lists a typical set of $\alpha^{\prime}, \beta^{\prime}$ values. The middle panel of Fig. 8 shows the results for the $\Delta \eta$ values obtained for these $\alpha^{\prime}, \beta^{\prime}$ combinations for each of the three solutions, with $\phi_{\text {off }}$ set to $18^{\circ}$. The mean value of $\Delta \eta$, computed from all the points across the pulse window (except the 4 outliers), is shown in Col. 5 of Table 2. As can be seen, these $\Delta \eta_{\text {avg }}$ values are very close to the expected values of $360^{\circ} / N_{\mathrm{sp}}$ (given in Col. 4 of the table). These results are very sensitive to small changes in values of $\alpha^{\prime}$ and $\beta^{\prime}$, with changes as small as $\sim 0.2$ (in any one of the angles) leading to substantial deviations in the $\Delta \eta$ curves. The solutions are also quite sensitive to the value of $\phi_{\text {off }}$, with small changes of the order of $10-15 \mathrm{deg}$ causing significant deviations from acceptable solutions. This is illustrated with the three sample results in the bottom panel in Fig. 8, which are computed for 3 different values of $\phi_{\text {off }}$. The best results are obtained for $\phi_{\text {off }}=18^{\circ}$, and the values in Table 2 are for this choice.

The solutions for $P_{3}$ and $P_{4}$ are given in Cols. 6 and 7 of Table 2, both in units of seconds and in units of the period, $P_{1}$. The results for the drift rate are in Col. 8. The percentage change in the amplitude of the drift rate to account for the largest typical deviation mentioned in Sect. 2 is given in Col. 9. The largest fractional change of $7.4 \%$ is needed for the case of $N_{\mathrm{sp}}, k=14,1$, and it reduces to $2.8 \%$ for the case of
$N_{\mathrm{sp}}, k=16,3$. Thus, the changes in the mean drift rate required to explain the observed drift rate variations are only a few percent.

\section{Discussion and summary}

1. On the location and arrangement of sparks in PSR B0826-34: Our analysis and interpretation of the data for PSR B0826-34 reveals that we are looking at a pulsar that is very close to being an aligned rotator, with a value of $\alpha$ less than $5^{\circ}$, and more likely to be around $1^{\circ}$ to $2^{\circ}$. Consequently, our line of sight traces out a circular track centred almost around the dipole magnetic axis. This not only gives the exceptionally wide profile that we observe for this pulsar, but also allows us to sample the emission pattern along a ring around the magnetic axis. This radiation pattern reveals the presence of multiple drifting bands of subpulses (up to 7 are visible), which we interpret as produced by subpulse producing plasma columns associated with multiple sparks circulating in the ring. This is the first pulsar for which direct evidence for such a large number of sparks has been obtained - all previous cases show evidence for at most 2-3 drift bands visible within the pulse window. Hence our results and interpretation can be considered as another direct confirmation of the idea of rings of circulating sparks on the polar cap, which on the average form conal beams of emission (Gil et al. 1993; Kijak \& Gil 2002).

The other interesting result is that the measured variation of $P_{2}$ - the longitude separation between the multiple driftbands - across the main pulse window is incommensurate with the values of $\alpha$ and $\beta$ for the dipole geometry that we infer from the variation of main pulse width with radio frequency and the known polarization angle variation. This leads us to a new and interesting conclusion: that the pattern of drifting subpulses does not circulate around the dipole magnetic axis. The actual point of circulation (which we refer to as the "local pole"), is determined to be only $\sim 3^{\circ}$ away from the dipole axis; however, this small difference is enough to cause significant differences in the observed values of $P_{2}$.

What determines the location of this centre of circulation and how do we understand this in the context of the existing theoretical models? In our interpretation, this centre of circulation of the drift pattern is a local pole produced by the more complicated magnetic field structure that could exist close to the neutron star surface. Though we believe that the neutron star magnetic field is practically dipolar at the heights at which the radio emission is generated in the magnetosphere, there is now a growing set of evidence that the magnetic field close to the neutron star surface (up to a few stellar radii) may have higher order multi-pole structure (see Urpin \& Gil 2004, for review). In fact, it could be a "sun-spot" like magnetic field (e.g., GS00). It is worth noting that the higher magnetic field strength and the much smaller radii of curvature that such a field configuration provides are actually essential for the working of the basic pair production mechanism inside the vacuum gap that drives the RS75 kind of models (Gil \& Melikidze 2002, and references therein). In such a scenario, it is possible to have on the polar cap, a local pole of the magnetic field configuration whose location is not aligned with that of the pole of the 
Table 2. Spark geometry and drift rate solutions for PSR B0826-34.

\begin{tabular}{cccccccccc}
\hline \hline$N_{\text {sp }}$ & $k$ & $\alpha^{\prime}, \beta^{\prime}$ & $\Delta \eta_{\exp }$ & $\Delta \eta_{\text {avg }}$ & $\begin{array}{c}P_{3} \\
(\mathrm{~s})\left(P_{1}\right)\end{array}$ & $\begin{array}{c}P_{4} \\
(\mathrm{~s})\left(P_{1}\right)\end{array}$ & $\begin{array}{c}D_{\mathrm{p}} \\
(\% / \mathrm{pulse})\end{array}$ & $\begin{array}{c}\Delta D_{\mathrm{p}} / D_{\mathrm{p}} \\
(\%)\end{array}$ & $\begin{array}{c}\mathrm{SF} \\
\left({ }^{\circ}\right)\end{array}$ \\
\hline 14 & 1 & $0.8,2.8$ & 25.7 & 25.64 & $1.850\left(1.00 P_{1}\right)$ & $25.90\left(14.0 P_{1}\right)$ & 25.7 & $<7.4$ & 0.36 \\
15 & 2 & $0.6,2.2$ & 24.0 & 24.06 & $0.925\left(0.50 P_{1}\right)$ & $21.95\left(7.50 P_{1}\right)$ & 48.0 & $<4.0$ & 0.67 \\
16 & 3 & $0.8,3.2$ & 22.5 & 22.52 & $0.617\left(0.33 P_{1}\right)$ & $9.86\left(5.33 P_{1}\right)$ & 67.5 & $<2.8$ & 0.94 \\
\hline
\end{tabular}

dipolar field. In models with vacuum gaps close to the neutron star surface, it will be the structure of this non-dipolar magnetic field that will control the activities in the vacuum gap. In the model of GS00, the location of this local pole is taken as the location of the central spark (anchored to the local pole) that constitutes the core beam of emission. The rest of the polar cap is then populated by spark discharges that are arranged in concentric circles around the central spark, leading to a nested cone structure for the emission beam. Within each circle, the sparks circulate due to the $\boldsymbol{E} \times \boldsymbol{B}$ drift in a manner similar to the original RS75 model. Though the higher order field dominates close to the neutron star surface, with increasing height it is thought to make a smooth transition to the dipolar field that dominates at higher altitudes. Thus we have a situation where the basic distribution of the seeds of emission (the sparks) on the polar cap is decided by the higher order surface magnetic field, while the actual height of the radio emission (and the evolution with frequency thereof) is dictated by the dipolar field at altitudes of typically about 50 stellar radii.

Our results and inferences thus lend strong support to this picture of strong non-dipolar magnetic fields close to the neutron star surface. Both theoretical and observational evidence is accumulating for the existence of such surface magentic field anomalies (for reviews see Geppert et al. 2003; and Urpin \& Gil 2004). What physical mechanism could create such smallscale (comparable with the polar cap radius) sun-spot like surface magnetic field structures? One possibility is the dynamo mechanism acting in the newborn neutron star. This idea was recently discussed by Urpin \& Gil (2004) who concluded from equipartition of magnetic energy and kinetic energy of the convective motion in a proto-netron star that a dynamo produces small-scale field structures with a strength of about $10^{13} \mathrm{G}$. However, this seems to be a quite speculative idea. The values of the electric conductivity, typical scale length and convective velocities used by Urpin \& Gil (2004) imply a magnetic Reynolds number many orders of magnitude larger than any imaginable critical value for a dynamo. Therefore, no reliable conclusion about the dynamo effect can be drawn without calculating growth rates by use of realistic velocity profiles. It seems that a more realistic scenario is provided by Geppert et al. (2003), who argued that the Hall-instability (Rheinhardt \& Geppert 2002) can create small-scale poloidal field structures from a sufficiently strong subsurface toroidal magnetic field. The geometrical structure of these small-scale anomalies resembles that of some types of "star-spots". The magnetic field strength in these anomalies should be about $10^{13}$ Gauss.
2. The two cone model for PSR B0826-34: For most of the work presented here, we have concentrated on the emission properties of the main pulse region of this pulsar. However, as we noted in Sect. 2, there is a weak interpulse seen in our $318 \mathrm{MHz}$ average profile, which becomes gradually stronger as we go to higher frequencies, and even dominates over the main pulse emission at frequencies higher than $\sim 1 \mathrm{GHz}$ (see Fig. 1). The natural explanation for this is emission from a second, outer conal ring with the geometry contriving such that the line of sight samples a large fraction of the inner ring at the lower frequencies $(\sim 300 \mathrm{MHz})$, while missing most of the outer ring. Then, as the emission beams reduce in angular size at the higher frequencies, the line of sight first comes closer to the inner ring (in the main pulse region) - at around $600 \mathrm{MHz}-$ and then actually crosses beyond the inner ring at frequencies above $1000 \mathrm{MHz}$, leading to relatively weak emission in the main pulse window. This evolution naturally explains the increased "filling-up" of the bridge region in the main pulse window as we go from $318 \mathrm{MHz}$ to $645 \mathrm{MHz}$. At the same time, the frequency evolution of the beams brings the outer conal ring closer to the line of sight in the interpulse region. This evolution is such that at around $600 \mathrm{MHz}$, significant part of the outer ring begins to be seen by the line of sight and at frequencies around $1.4 \mathrm{GHz}$, the line of sight is actually grazing along the outer ring in the interpulse region.

We have modeled this configuration of two conal rings using a simulation program that takes a given distribution of sparks on the polar cap and translates them along dipolar field lines to frequency dependent heights of emission in the magnetosphere and generates the observed pulse profiles that would be seen along a specified line of sight (see Appendix B for details). The dashed lines in Fig. 1 show the simulated profiles obtained for a model having two conal rings of emission with frequency dependent radii determined by Eq. (A.3) and centred around the local pole which is located at the point of the polar cap characterized by $\alpha^{\prime}=0.44, \beta^{\prime}=3.06$ and $\Phi_{\text {off }}=17.3$. As can be seen, the match with the observed profiles is quite good, providing direct support for the model of 2 conal rings. One important point worth stressing is that once the amplitude ratio of main pulse to interpulse is fixed at one frequency, then the spectral evolution of these profile components is almost entirely due to dipolar spreading of magnetic field lines in the emission region.

Our two conal ring model for PSR B0826-34 makes clear predictions about how the average profile and single pulse characteristics should evolve with frequency and suitable experiments can be devised for testing these. For example, one of the interesting things that it predicts is that the position angle curve 
should be shifted with respect to the centre of the main pulse window, because of the $\phi_{\text {off }}$ rotational phase difference between the fiducial planes containing the local pole axis and the dipole axis. In Fig. 6 of Biggs et al. (1985), there is some hint that the centre of the PA swing occurs at slightly later longitudes than the centroid of the main pulse, but the quality of the data is not good enough to ascertain this. Clearly, good quality polarimetric observations of PSR B0826-34 can help in testing this aspect of our model. Further, since our model predicts the interpulse emission region to arise from a different conal ring, we expect that the subpulse properties here could be different from those for the main pulse region. For example, depending on the number of sparks populating the outer ring, the measured value of $P_{2}$ could be very different from the value we measure for the inner ring. Perhaps the most interesting feature would be the value of drift rate and inferred values of $P_{3}$ and $P_{4}$ in the interpulse region at 600 to $1400 \mathrm{MHz}$.

3. Pulse profile classification of PSR B0826-34: It is clear from our work that there is a need for a revision of the earlier classification of PSR B0826-34 as a "M-type" pulsar, i.e. a pulsar where the line-of-sight and emission geometry are such that multiple cones of emission are visible along with the central core component (e.g., Rankin 1993; Lyne \& Manchester 1988). In our model, all the emission seen in the main pulse window originates from a single conal ring of emission and that seen in the interpulse regions comes from the second, outer conal ring of emission. The line of sight thus does not sample the central core region at all, making this a completely conal pulsar. Though the average profiles of this pulsar sometimes tend to show partial evidence for discrete emission components in the saddle region between the leading and trailing emission peaks in the main pulse region (as can be seen in Fig. 1), we believe these are artefacts of the quasi-longitude stationary drift pattern of the multiple drift bands, especially when average profiles are made over relatively short intervals of time, from limited number of pulses.

The classification of PSR B0826-34 as a "M-type" profile pulsar seemed strongly supported by the prominent circular polarization, reversing sense in the central part of the profile (Biggs et al. 1985, their Fig. 6). It therefore follows from our analysis of drifting subpulse patterns in this pulsar, that sense reversing circular polarization does not have to be associated with core emission only. It can also appear in conal components, if the related subpulses (as in Fig. 3) are quasi-stationary in phase (for more details see Gil et al. 1995).

\section{Inferences from the drift behaviour of PSR B0826-34:}

We now discuss the results for the drifting behaviour of this pulsar in some detail. Not only do we see subpulse emission from several spark-associated plasma columns, we are also able to detect the presence of correlated drift pattern from all of these subpulses. The observed apparent reversals of drift direction, which are difficult to explain under any physical model of the subpulse drift phenomenon, are naturally explained as a combination of aliasing of the true drift rate coupled with small $(\approx 3-8 \%)$ variations of the true drift rate. Thus, in our interpretation, the intrinsic drift rate does not show any reversals in sign, but only small changes in amplitude, making the behaviour of this pulsar compatible with these models. The inferred values of both the mean drift rate and the proposed small variations are worthy of a detailed discussion, which we now present.

As we have shown, a unique solution for the spark and emission geometries for PSR B0826-34 is not possible with the current data, as the alias order and $N_{\mathrm{sp}}$ can not be unambiguously determined. Instead, for a given choice of the value of one of these, the value for the other that yields acceptable solutions can be found. The values for $D, P_{3}$ and $P_{4}$ can then be found for these cases and compared with expectations from models. These results are summarised in Table 2, for three choices of the value of $N_{\text {sp }}$. The drift rates for most of the solutions are much lower than the values expected for the pure vacuum gap models (e.g., RS75; GMG03). Correspondingly, the values of $P_{4}$ are substantially longer than the prediction. This result for PSR B0826-34 is similar to the results obtained for PSR B0943+10 (Deshpande \& Rankin 1999) and PSR B0809+74 (van Leeuwen et al. 2003). A natural explanation for this behaviour is provided by GMG03 who show that if the $\boldsymbol{E} \times \boldsymbol{B}$ drift is considered in a non-ideal vacuum gap, i.e. one in which there is a finite amount of charge flow (e.g., Cheng \& Ruderman 1980), then the predicted value of the drift rate is reduced. The proposed agency for the control of the ion flow in the gap is thermal regulation, i.e. the ratio of the surface temperature to the ion critical temperature controls the rate of ion flow, and hence the electrical potential drop across the gap and hence the value for the $\boldsymbol{E} \times \boldsymbol{B}$ drift rate. This effect is characterised by a "shielding factor" by GMG03 who find typical values for it to be $\sim 0.2$. For our possible solutions, the values of the shielding factor (SF) are shown in Col. 10 of Table 2. For the slowest drift rate solution, this factor is 0.36 and it increases to 0.94 (i.e. close to vacuum gap conditions) for the case of $N_{\text {sp }}, k=16,3$. From this it is clear that solutions for $k>3$ are not viable as the implied drift rate would be too fast - more than the maximum that is produced under pure vacuum gap conditions.

In the above scenario, the small variations around the mean drift rate would then be due to small changes in the neutron star's surface temperature in the polar cap region. For example, to produce a $8 \%$ change in the drift rate, we find, using the formulation in GMG03, that only a $0.14 \%$ change in the surface temperature is needed. This means a change of about $3500 \mathrm{~K}$ out of a mean temperature of $\sim 2.5 \times 10^{6} \mathrm{~K}$. What could case such small and cyclic (though quasi-periodic at best) variations in the polar cap temperature is an interesting and open question. One possibility is a kind of "self-regulating" mechanism of heating and cooling of the polar cap by increased and reduced spark activities. Whatever it maybe, from our earlier deductions (at the end of Sect. 3.2.1) about the regions of slower and faster than normal drift rates, it would imply that the process of cooling below the mean temperature (which would lead to a faster than mean drift rate) is observed to be a relatively slower and smoother process than the reverse process of heating (associated with slower than mean drift rates), which is found to be more abrupt and faster. Further, it is interesting 
that the observed drift rate fluctuations are found to be highly correlated for all the visible drift bands (Sect. 2), implying that the small temperature variations (if they are the cause of the drift fluctuations) occur simultaneously on a fairly large scale across a large part of the polar cap. Here, detection and comparison of subpulse drift (and its variations) in the interpulse region (which we believe is due a second ring of sparks on the polar cap) with that in the main pulse region should produce valuable additional insight.

It is worth noting that in a relatively old and isolated pulsar like B0826-34 with a characteristic age about $3 \times 10^{7}$ years (see Sect. 1), the standard cooling scenario predicts a rather low surface temperature of about $4 \times 10^{4} \mathrm{~K}$ (see Fig. 5 in Page et al. 2000). Thus, an effective heating mechanism must exist to raise the polar cap temperature over 50 times. Self-consistent calculations performed by Gil et al. (2003) for this pulsar (see their Sect. 4.4.2) show that the bombardment of the polar cap by backflowing electrons from subpulse associated sparks heat the polar cap area to temperatures $\sim 2.5 \times 10^{6} \mathrm{~K}$. It is worth mentioning that the observational estimates of the polar cap temperatures based on first results from XMM satellite indicate values above $10^{6} \mathrm{~K}$ in a number of pulsars (Becker \& Aschenbach 2002).

\section{General implications for the average pulsar population:} Finally, we turn to a discussion of the implications of slow variations of drift rate for the average pulsar population. If the few percent variation of drift rate that we infer for PSR B0826-34 is a generic phenomenon affecting a large number of pulsars, then it could explain some of the mysteries of non-detection of periodic drift variations in the fluctuation spectra analysis of several pulsars. Depending on the time scale of the drift rate fluctuations, compared to the pulsar period and the length of data taken for fluctuation spectra analysis, it is possible that drift rate variations could significantly, or even completely, blur the periodic signal. Thus it is possible that several pulsars have drifting subpulses, but with some fluctuations in the drift rate, leading to reduced chances of detection of the phenomenon in a traditional fluctuation spectrum analysis. Even amongst the "classical" drifting pulsars (e.g. PSR B0031-07, PSR B0943+10), variations in drift rate are a common observed feature.

As a specific example, we would like to point out the case of PSR B0540+23, which almost certainly shows stroboscopic phenomena due to aliasing, similar to PSR B0826-34. As reported by Nowakowski (1991), the subpulse drift is irregular, without specified drift direction and with different speeds, including zero speed. As a result, there is no specified value of $P_{3}$, and there is no signature in the fluctuation spectrum. Therefore, this pulsar is very similar to PSR 0826-34 (see Figs. 4-6 in Nowakowski 1991), except that the pulse profile is much narrower, implying a larger inclination angle.

6. Summary: Using results from sensitive single pulse observations with the GMRT at $318 \mathrm{MHz}$, we have made significant progress in unraveling the complex drifting subpulse pattern of PSR B0826-34. In the process, we have shown that this pulsar is very close to being an aligned rotator. This explains the unusually wide profile and multiple drift bands seen for this pulsar. We have modeled the observed profiles and shown evidence for the presence of two conal rings of emission. A detailed treatment of effects of aliasing and geometry on the drifting subpulse pattern has been provided. We have given an explanation for the observed variability of the drift rate, including the apparent reversals of drift direction, within the framework of the currently available theoretical models. As a result, we have shown that small variations in the surface temperature of the neutron star polar cap can explain the observed drift pattern. Finally, we have also demonstrated that the subpulse pattern shows evidence for the presence of non-dipolar magnetic fields close to the neutron star surface. Clearly, there is a lot to be learnt from PSR B0826-34.

Acknowledgements. We thank the staff of the GMRT for help with the observations. The GMRT is run by the National Centre for Radio Astrophysics of the Tata Institute of Fundamental Research. Y.G. would like to acknowledge the support from the Institute of Astronomy, University of Zielona Gora, for a short sabbatical visit during which this work was initiated. This paper is supported in part by the Grant 1 P03D 02926 of the Polish State Committee for Scientific Research. We thank E. Gil for technical help and R. Nityananda for a critical reading of the manuscript.

\section{Appendix A: Pulsar emission geometry formulae}

The geometry of pulsar radiation is described by Manchester $\&$ Taylor (1977, see their Fig. 10.4). At the rotational phase $\phi$, the angle between the observer's line of sight and the dipole magnetic axis is given by

$\cos \Gamma=\cos \alpha \cos \zeta+\sin \alpha \sin \zeta \cos \phi$,

where $\zeta=\alpha+\beta, \alpha$ is the angle between the rotation and the magnetic axes and $\beta$ is the impact angle of the closest approach of the line of sight to the magnetic axis.

The angle $\Gamma$ expressed above is also the angle between the dipole axis and the tangent to magnetic field lines at points where the emission observed at the rotational phase $\phi$ originates. Using equations for the dipolar field lines, this opening angle (also referred to as the beam radius) can be described as

$\rho=1.24 s\left[r_{\mathrm{em}}(v) / R\right]^{1 / 2} P_{1}^{-1 / 2}$,

where $P_{1}$ is the pulsar period, $r_{\mathrm{em}}(v)$ is the emission altitude (at which radiation at a given frequency $v$ is generated) and $R=10^{6} \mathrm{~cm}$ is the neutron star radius. The mapping parameter $0 \leq s=d / r_{\mathrm{p}} \leq 1$ is determined by the locus of dipolar field lines on the polar cap ( $s=0$ at the pole and $s=1$ at the polar cap edge), where $d$ is the distance from the magnetic axis to the field line on the polar cap corresponding to a certain detail of the pulse profile and $r_{\mathrm{p}}=1.4 \times 10^{4} P_{1}^{-1 / 2} \mathrm{~cm}$ is the canonical polar cap radius.

According to the generally accepted concept of radius-tofrequency mapping, higher frequencies are emitted at lower altitudes than lower frequencies. The emission altitude can be described by (Kijak \& Gil 2003, and references therein)

$r_{\mathrm{em}}=(40 \pm 8) R v_{\mathrm{GHz}}^{-0.26 \pm 0.09} \dot{P}_{-15}^{0.07 \pm 0.03} P_{1}^{0.30 \pm 0.05}$, 
where $R=10^{6} \mathrm{~cm}, v_{\mathrm{GHz}}$ is the observing frequency (in $\mathrm{GHz}$ ), $P_{1}$ is the pulsar period (in seconds) and $\dot{P}_{-15}$ is the period derivative in units of $10^{-15} \mathrm{~s} / \mathrm{s}$.

The polarization position angle (PA) curve is described by

$\tan \psi=\frac{\sin \alpha \sin \phi}{\sin \zeta \cos \alpha-\cos \zeta \cos \phi} ;$

and the value of the steepest gradient of the PA curve is given by

$\left.\frac{\mathrm{d} \psi}{\mathrm{d} \phi}\right|_{\max }=\frac{\sin \alpha}{\sin \beta}$.

At a given frequency, $v_{\mathrm{GHz}}$, for a chosen combination of $\alpha, \beta$ and $s$, the value of the beam radius, $\rho$, can be found using Eqs. (A.3) and (A.2). Then, using $\Gamma(\phi)=\rho(v)$, the profile width corresponding to the emission peaks of the conal components, $2 \phi$, can be found from Eq. (A.1) and compared with observed values. For a chosen combination of $\alpha$ and $\beta$, the steepest gradient can be computed using Eq. (A.5) and compared with observations; further, using the estimate of $\phi$, the total swing of the polarization angle across the pulse, $\Delta \psi$, can be computed from Eq. (A.4) and compared with observed data. Combinations of $\alpha, \beta$ and $s$ that meet all these criteria for all the frequencies listed in Table 1 were found by doing a grid search in the values of $\alpha, \beta$ and $s$.

\section{Appendix B: Simulations of aliasing effects}

To illustrate the effects of aliasing in the subpulse drift patterns of PSR B0826-34, we simulate the radiation of this pulsar, assuming that its single pulse structure reflects the circumferential motion of 14 sparks at a distance $d=0.44 r_{\mathrm{p}}=33 \mathrm{~m}$ from the pole. For the emission geometry we adopted $\alpha=2.5$ and $\beta=1.0$, and an emission altitude for the frequency of $318 \mathrm{MHz}$ was estimated from Eq. (A.3). We use the simulation technique described in GS00 (and references therein). Each spark is modeled as a circular entity with a characteristic dimension equal to the height $h$ of the quasi-steady acceleration gap formed above the polar cap. The adjacent sparks are separated from each other also by about $h$ (spark centers are separated by $2 h$ ). Since the elementary pulsar radiation is relativistically beamed along dipolar magnetic field lines, the spark-associated intensity pattern can be transformed from the polar cap to the emission region and then to the observer according to a given geometry $\left(\alpha, \beta, r_{\mathrm{em}}\right)$. The spark-associated subbeams, related to subpulses observed in single pulses, perform a circumferential motion due to the $\boldsymbol{E} \times \boldsymbol{B}$ drift of the spark plasma. The simulation program allows for circulation around either the magnetic dipole or local pole of surface magnetic field (assuming that this surface field is also axially symmetric on the polar cap), with a desired angular drift rate, $D=360^{\circ} / P_{4}$, where $P_{4}$ is the period of spark circulation.

For given values of $P, \dot{P}, \alpha$ and $\beta$ the number of subpulses and their phases in a single pulse are determined by the value of $D_{p}$. We performed an experiment with time varying drift rates, starting with $D_{p}=0$ and incrementing it by 0.03 every rotational period $P_{1}$ (this increment was chosen to make sure that cycles of gradual variations of the drift rate have duration of about $100 P_{1}$, as observed in real data). The idea was to find a sequence of about 100 pulses with varying $D_{p}$ in a range appropriate to produce curved drift-bands similar to those visible in Fig. 3. A sample result of our simulations is presented in Fig. 9, with all the relevant information provided in the top panel and on both sides of the pulse window. The last column indicates the actual value of $D_{p}=0.03 n$ per period, where $n$ is the rotational period number indicated on the vertical axis. Next to $D_{p}$ we list values of $P_{4} / P_{1}=360^{\circ} / D_{p}$. On the other side of the pulse window we give values of $P_{3} / P_{1}=P_{4} /\left(14 P_{1}\right)$ and the column just before shows the fluctuation frequency, $f_{3} P_{1}=P_{1} / P_{3}$.

What can we learn from simulated patterns presented in Fig. 9? First of all, pulses around $n=1$ show that were there no $\operatorname{drift}\left(D_{p}=0\right)$, the observer would clearly see 7 out of 14 sparks in the form of longitude stationary subpulses. As the drift rate increases with the increasing number of periods, the subpulse drift with time varying rate becomes more and more apparent. However, up to about $n=100$, the subpulse drift is relatively slow, non-aliased and proceeds from the leading to the trailing edge of the profile. This is the real drift direction and the observed drift-bands are formed by the same sparks. This is, however, not true in the region well above $n=100$, where all kinds of stroboscopic aliasing effects become visible. We have marked regions where the apparent drift-bands are formed by subpulses appearing at approximately the same phase every $m$-th period, where $m=5,4,3,2$ and so on. It is worth noting that the number of apparent drift-bands is about $7 \mathrm{~m}$. The drift-bands change the apparent drift direction due to the aliasing effect, every time $f_{3}$ crosses a multiple of the Nyquist frequency.

Obviously, the region below $n=800$ does not correspond to drifting subpulses in PSR 0826-34, because it shows alternating (even-odd), longitude stationary intensity modulations, which are not observed in this pulsar. It seems, however, that its drifting subpulse patterns are well modeled by the region between $n=800$ and $n=900$, which could represent one cycle of multiple curved drift-bands visible in Fig. 3. A clear pattern of seven drift-bands is visible, moving in an aliased direction (from the trailing to the leading edge) in the first half of the cycle, and in the true direction (from the leading to the trailing edge) in the second half of the cycle. The drift direction change occurs at $f_{3}=1 / P_{1}$ (or $P_{3}=P_{1}$ ), which is twice the conventional Nyquist frequency. At this stage the pattern advances exactly by one subbeam per one pulsar period $P_{1}$ and the apparent drift-bands are formed by successive adjacent subbeams. The corresponding values of $P_{4} / P_{1}=14$ and $D_{p}=25^{\circ} 7$ per period. However, 50 pulses earlier, at the beginning of a cycle, $P_{4} / P_{1}=14.4$ and $D_{p}=25^{\circ}$ per period, while about 50 pulses later, at the end of a cycle, $P_{4} / P_{1}=13.3$ and $D_{p}=27^{\circ} .1 \mathrm{per}$ period. This means that the pattern speeds up along each cycle, increasing $D_{p}$ by about $8 \%$.

Although we present simulations only for the case of 14 sparks, the qualitative results are very similar for other values of $N_{\mathrm{sp}}$. Results of simulations for 15 and 16 sparks, as well as those for 14 sparks presented in Fig. 9, agree exactly with the drift rate solutions presented in Table 2 . 


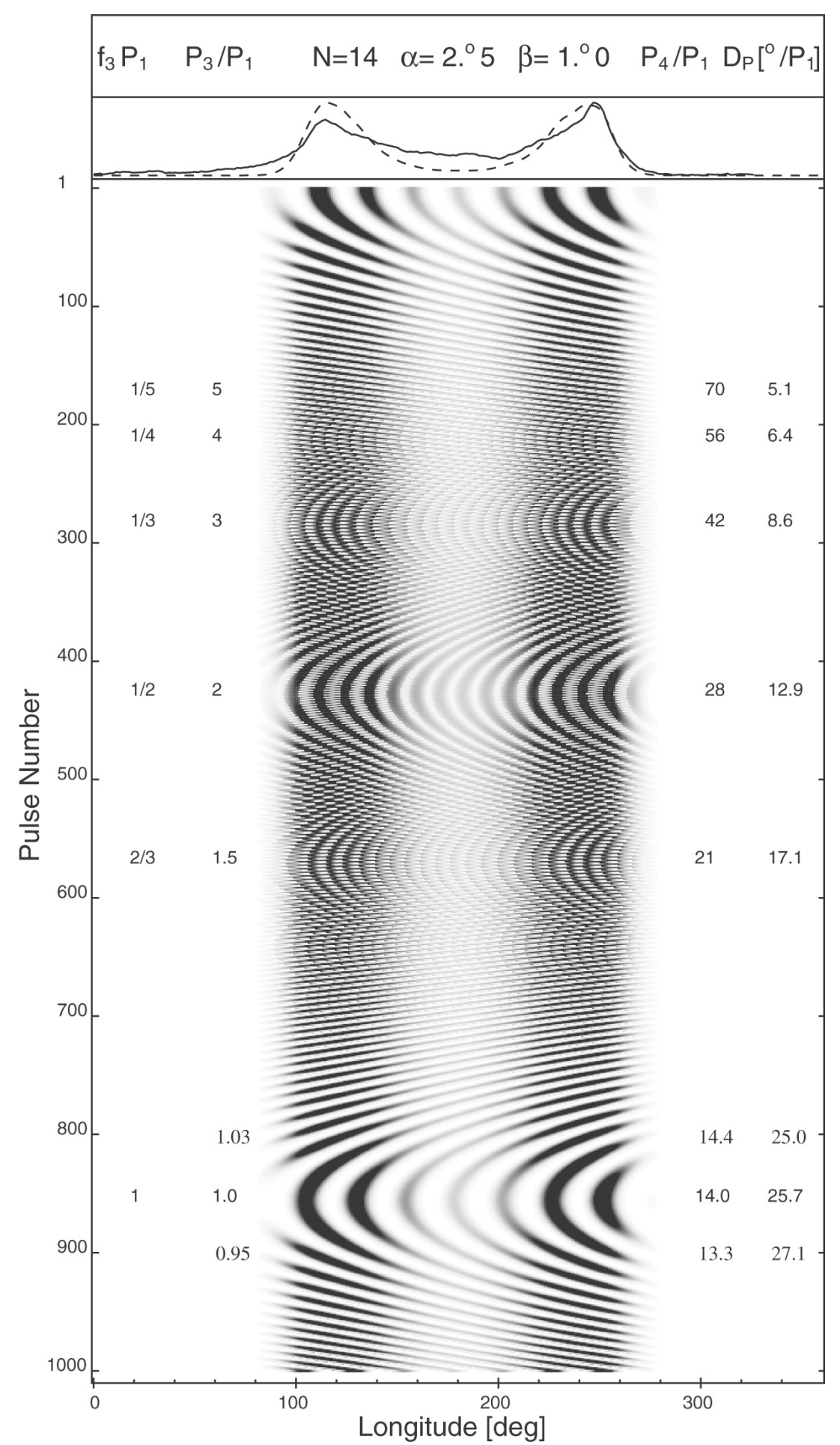

Fig. 9. Simulations of the subpulse drift phenomenon with time varying drift rate $D_{p}(n)=0.03 n$ per period, where $n$ is the number of pulsar periods shown on the vertical axis (see text for explanation of other symbols). The quasi-periodic patterns of drifting subpulses observed in PSR B0826-34 (Fig. 3) are well modeled by the sector between $n=800$ and $n=900$, with $D_{p}$ increasing from $25^{\circ}$ to $27^{\circ} .1$ per period.

\section{References}

Allen, M. C., \& Melrose, D. B. 1983, Proc. Astr. Soc. Aust., 5, 191 Backer, D. B. 1973, ApJ, 182, 245

Becker, W., \& Aschenbach, B. 2002, Proc. of 270 WE - Heracus Seminar, MPG Rep. 278, 64

Biggs, J. D., McCulloch, P. M., Hamilton, P. A., Manchester, R. N., \& Lyne, A. G. 1985, MNRAS, 215, 281
Cheng, A. F., \& Ruderman, M. A. 1980, ApJ, 235, 576

Drake, F. D., \& Craft, H. D., Jr. 1968, Nature, 220, 231

Deshpande, A. A., \& Rankin, J. M. 1999, ApJ, 524, 1008

Durdin, J. M., Large, M. I., Little, A. G., et al. 1979, MNRAS, 186, 39

Geppert, U., Rheinhardt, M., \& Gil, J. 2003, A\&A, 412, L33

Gil, J., Kijak, J., \& Seiradakis, J. H. 1993, A\&A, 272, 268 
Gil, J., Kijak, J., Maron, O., \& Sendyk, M. 1995, A\&A, 301, 177

Gil, J., \& Melikidze, G. I. 2002, ApJ, 577, 909

Gil, J. A., Melikidze, G. I., \& Mitra, D. 2002, A\&A, 388, 246

Gil, J., Melikidze, G. I., \& Geppert, U. 2003, A\&A, 407, 315 (GMG03)

Gil, J., \& Sendyk, M. 2000, ApJ, 541, 351 (GS00)

Gupta, Y., Gothoskar, P. B., Joshi, B. C., et al. 2000, in Pulsar Astronomy - 2000 and Beyond, ed. M. Kramer, N. Wex, \& R. Wielebinski, IAU Coll., 177, ASP Conf. Ser., 105, 277

Jones, P. B. 1984, MNRAS, 209, 569

Kazbegi, A., Machabeli, G., Melikidze, G., \& Shukre, C. 1996, A\&A, 309,515

Kijak, J., \& Gil, J. 2002, A\&A, 392, 189

Kijak, J., \& Gil, J. 2003, A\&A, 397, 969

Lyne, A. G., \& Manchester, R. N. 1988, MNRAS, 234, 477

Manchester, R. N., \& Taylor, J. H. 1977, Pulsars (San Francisco: Freeman)

Miller, M. C., \& Hamilton, R. J. 1993, ApJ, 411, 298

Narayan, R., \& Vivekanand, M. 1983, A\&A, 113, L3
Nowakowski, L. A. 1991, ApJ, 377, 581

Page, D., Geppert, U., \& Zannias, T. 2000, A\&A, 360, 1052

Rankin, J. M. 1983, ApJ, 274, 333

Rankin, J. M. 1986, ApJ, 301, 901

Rankin, J. M. 1993, ApJS, 85, 145

Rheinhardt, M., \& Geppert, U. 2002, Phys. Rev. Lett., 88, 101103

Reyes, F., Aparici, J., \& Olmos, F. 1995, A\&A, 301, 182

Ruderman, M. A., \& Sutherland, P. G. 1975, ApJ, 196, 51 (RS75)

Ruderman, M. 1976, ApJ, 203, 206

Taylor, J. H., Manchester, R. N., \& Huguenin, G. R. 1975, ApJ, 195, 513

Turner, P. 1980, Ph.D. Thesis, University of Tasmania

Urpin, V., \& Gil, J. 2004, A\&A, 415, 305

van Leeuwen, A. G. J., Stappers, B. W., Ramachandran, R., \& Rankin, J. M. 2003, A\&A, 399, 223

van Ommen, T. D., D’Alessandro, F., Hamilton, P. A., \& McCulloch, P. M. 1997, ApJ, 287, 307

Wright, G. A. E. 2003, MNRAS, 344, 1041 